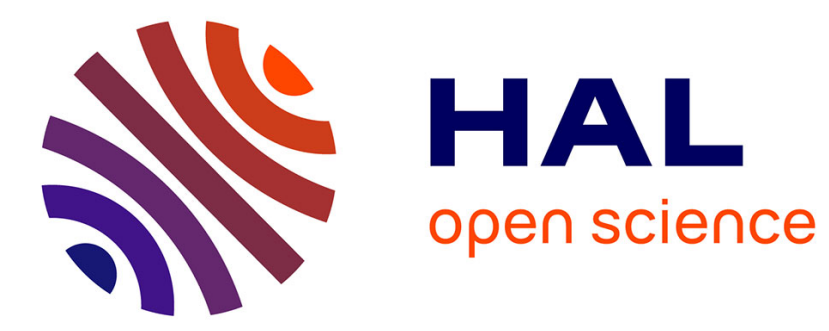

\title{
Time-domain study of the Drude-Born-Fedorov model for a class of heterogeneous chiral materials
}

\author{
Patrick Ciarlet, Guillaume Legendre, Serge Nicaise
}

\section{To cite this version:}

Patrick Ciarlet, Guillaume Legendre, Serge Nicaise. Time-domain study of the Drude-Born-Fedorov model for a class of heterogeneous chiral materials. 2011. hal-00430196v2

\section{HAL Id: hal-00430196 \\ https://hal.science/hal-00430196v2}

Preprint submitted on 5 Jan 2011

HAL is a multi-disciplinary open access archive for the deposit and dissemination of scientific research documents, whether they are published or not. The documents may come from teaching and research institutions in France or abroad, or from public or private research centers.
L'archive ouverte pluridisciplinaire HAL, est destinée au dépôt et à la diffusion de documents scientifiques de niveau recherche, publiés ou non, émanant des établissements d'enseignement et de recherche français ou étrangers, des laboratoires publics ou privés. 


\title{
TIME-DOMAIN STUDY OF THE DRUDE-BORN-FEDOROV MODEL FOR A CLASS OF HETEROGENEOUS CHIRAL MATERIALS
}

\author{
PATRICK CIARLET, JR. $\dagger$ GUILLAUME LEGENDRE ${ }^{\ddagger}$ AND SERGE NICAISE§
}

\begin{abstract}
We deal with the well-posedness of the transient Maxwell equations in a particular class of heterogeneous isotropic chiral material modeled by the Drude-Born-Fedorov constitutive relations. A new formulation of the underlying evolution problem allows us to correct a previous result establishing the existence and uniqueness of the electromagnetic fields in a homogeneous medium.
\end{abstract}

1. Introduction. Chiral materials are examples of media which respond with both electric and magnetic polarization to either electric or magnetic excitations. Because of this peculiar behavior, they have been studied extensively by the electromagnetics community, for a wide range of applications, including prospective ones.

In this work, we consider the time-dependent Maxwell equations in a heterogeneous, isotropic chiral medium filling a bounded domain surrounded by a perfect conductor. Although their use is mainly restricted to timeharmonic applications, the Drude-Born-Fedorov constitutive relations (see for instance [10]) are used to model the behavior of the chiral material, within the scope of what is referred to in the literature as the optical response approximation [9]. Recent mathematical investigations dealing with electromagnetic waves in chiral media and involving the Drude-Born-Fedorov relations are the main topics of a number of articles, e.g. $[1,3]$ and references therein for the time-harmonic case, $[8,11]$ and references therein for the time domain case. Here, the well-posedness result erroneously stated in [8] is reexamined (and corrected) through a different mathematical interpretation of the evolution system in the case where the electric permittivity and magnetic permeability of the medium are both possibly non-constant and proportional to the chiral admittance. Our main result asserts the existence of a unique solution to the problem under a spectral condition involving the admittance. Its proof is based on the invertibility property of the so-called Drude-Born-Fedorov operator, some characterizations of the orthogonal of its range and the introduction of an appropriate invariant subspace of its inverse.

More precisely, we consider the following problem. Let $\Omega$ be a bounded subset of $\mathbb{R}^{3}$ with a Lipschitz boundary $\Gamma$, no assumption being made on the simple connectedness of $\Omega$, nor on the connectedness of $\Gamma$. We consider the time-dependent Maxwell equations, supplemented with boundary and initial conditions to close the system (the reader is referred to [8] for a justification of this model),

$$
\left\{\begin{array}{l}
\frac{\partial \boldsymbol{D}}{\partial t}-\operatorname{curl} \boldsymbol{H}=\mathbf{0} \text { in } \Omega \times(0, \infty) \\
\frac{\partial \boldsymbol{B}}{\partial t}+\operatorname{curl} \boldsymbol{E}=\mathbf{0} \text { in } \Omega \times(0, \infty) \\
\boldsymbol{H} \wedge \boldsymbol{n}=\boldsymbol{J}, \boldsymbol{E} \wedge \boldsymbol{n}=\mathbf{0} \text { on } \Gamma \times(0, \infty) \\
\boldsymbol{E}(0)=\boldsymbol{E}_{0}, \boldsymbol{H}(0)=\boldsymbol{H}_{0} \text { in } \Omega
\end{array}\right.
$$

subject to the Drude-Born-Fedorov constitutive relations

$$
\boldsymbol{D}=\varepsilon(\boldsymbol{E}+\beta \operatorname{curl} \boldsymbol{E}) \text { and } \boldsymbol{B}=\mu(\boldsymbol{H}+\beta \operatorname{curl} \boldsymbol{H}) .
$$

In the above equations, $\boldsymbol{D}$ is the electric displacement, $\boldsymbol{H}$ is the magnetic field, $\boldsymbol{B}$ is the magnetic induction, and $\boldsymbol{E}$ is the electric field. The vector $\boldsymbol{n}$ denotes the unit outward normal vector to the boundary $\Gamma$ and the field $\boldsymbol{J}$ is a surface current density flowing tangentially to the boundary. The positive scalars $\varepsilon, \mu$ and $\beta$ denote respectively the electric permittivity, the magnetic permeability, and the chirality admittance of the heterogeneous isotropic chiral material. It is assumed that these coefficients are real-valued bounded functions and uniformly positive definite, i.e. there exists constants $\varepsilon_{*}, \varepsilon^{*}, \mu_{*}, \mu^{*}, \beta_{*}$ and $\beta^{*}$ such that

$$
0<\varepsilon_{*} \leq \varepsilon(\boldsymbol{x}) \leq \varepsilon^{*}<+\infty, 0<\mu_{*} \leq \mu(\boldsymbol{x}) \leq \mu^{*}<+\infty
$$

$$
\text { and } 0<\beta_{*} \leq \beta(\boldsymbol{x}) \leq \beta^{*}<+\infty, \forall \boldsymbol{x} \in \bar{\Omega} \text {. }
$$

†POEMS, UMR 7231 CNRS/ENSTA/INRIA, École Nationale Supérieure de Techniques Avancées, 32, boulevard Victor, 75739 Paris cedex 15, France (patrick.ciarlet@ensta.fr).

${ }^{\ddagger}$ CEREMADE, UMR CNRS 7534, Université de Paris-Dauphine, Place du Maréchal De Lattre De Tassigny, 75775 Paris cedex 16, France. (guillaume.legendre@ceremade.dauphine.fr).

${ }^{\S}$ LAMAV, EA 4015, Université de Valenciennes et du Hainaut-Cambrésis, Le Mont Houy, 59313 Valenciennes cedex 9 , France (serge.nicaise@univ-valenciennes.fr). 
In addition, the class of chiral material considered in this paper is characterized by two more hypotheses: we require that there exist two constants $\kappa_{\varepsilon}$ and $\kappa_{\mu}$ such that

$$
\kappa_{\varepsilon}=(\varepsilon \beta)^{-1} \text { and } \kappa_{\mu}=(\mu \beta)^{-1} \text { in } \bar{\Omega},
$$

and moreover that the chiral admittance $\beta$ is piecewise regular, in the following sense: we suppose there exists a partition of $\Omega$ in a finite set of Lipschitz domains $\Omega_{1}, \ldots, \Omega_{K}$ such that the restriction of $\beta$ to each $\Omega_{k}, 1 \leq k \leq K$, belongs to $\mathscr{C}^{0}\left(\bar{\Omega}_{k}\right)$. Note that these conditions always hold if $\varepsilon, \mu$ and $\beta$ are constant, which is the case originally treated in [8]. On the other hand, when these coefficients are all piecewise constant, conditions (1.3) are satisfied if their respective jumps hold on the same interfaces. Physically speaking, we do not claim that this model is pertinent. However, from a mathematical point of view, these assumptions allow one to partly recover a familiar functional setting (see the discussion in subsection 7.2 in [8]), which is the one for Maxwell's equations in a nonhomogeneous, isotropic dielectric material.

We finally recall that the surface current density is linked to the surface charge density $\sigma$ by the charge conservation equation

$$
\frac{\partial \sigma}{\partial t}+\operatorname{div}_{\tau} \boldsymbol{J}=0 \text { on } \Gamma,
$$

in which $\operatorname{div}_{\tau}$ is the surface divergence operator. In the remainder of the paper, we restrict ourselves to a zero surface charge density, therefore the surface current density will be (surface) divergence free.

An outline of the paper is the following. In Section 2, we first introduce the various ingredients needed to set a proper mathematical framework for the physical problem (1.1)-(1.2). Section 3 is devoted to the invertibility of the operator underlying the Drude-Born-Fedorov constitutive relations (1.2). This intermediary result then allows us to establish the well-posedness of the evolution problem in the next section. Finally, we discuss the range of applicability of this result in section 5 .

2. Some definitions and technical results. Let us give some definitions and recall a number of results relative to the functional frawework used throughout the paper.

We first define the Hilbert spaces

$$
\begin{aligned}
& H(\operatorname{curl} ; \Omega)=\left\{\boldsymbol{v} \in L^{2}(\Omega)^{3} \mid \operatorname{curl} \boldsymbol{v} \in L^{2}(\Omega)^{3}\right\}, \\
& H\left(\operatorname{div} \beta^{-1} ; \Omega\right)=\left\{\boldsymbol{v} \in L^{2}(\Omega)^{3} \mid \operatorname{div}\left(\beta^{-1} \boldsymbol{v}\right) \in L^{2}(\Omega)\right\},
\end{aligned}
$$

equipped with their usual norms, and their respective, closed, subspaces

$$
\begin{aligned}
& H_{0}(\operatorname{curl} ; \Omega)=\{\boldsymbol{v} \in H(\operatorname{curl} ; \Omega) \mid \boldsymbol{v} \wedge \boldsymbol{n}=\mathbf{0} \text { on } \Gamma\} \\
& H_{0}\left(\operatorname{div} \beta^{-1} ; \Omega\right)=\left\{\boldsymbol{v} \in H\left(\operatorname{div} \beta^{-1} ; \Omega\right) \mid \beta^{-1} \boldsymbol{v} \cdot \boldsymbol{n}=0 \text { on } \Gamma\right\} \\
& H\left(\operatorname{div} \beta^{-1} 0 ; \Omega\right)=\left\{\boldsymbol{v} \in H\left(\operatorname{div} \beta^{-1} ; \Omega\right) \mid \operatorname{div}\left(\beta^{-1} \boldsymbol{v}\right)=0 \text { in } \Omega\right\}
\end{aligned}
$$

We also introduce the space $X\left(\Omega, \beta^{-1}\right)=H(\operatorname{curl} ; \Omega) \cap H\left(\operatorname{div} \beta^{-1} ; \Omega\right)$, and its subspaces $X_{N}\left(\Omega, \beta^{-1}\right)=$ $H_{0}(\operatorname{curl} ; \Omega) \cap H\left(\operatorname{div} \beta^{-1} ; \Omega\right)$ and $X_{T}\left(\Omega, \beta^{-1}\right)=H(\operatorname{curl} ; \Omega) \cap H_{0}\left(\operatorname{div} \beta^{-1} ; \Omega\right)$. It is well-known that the embedding of $X\left(\Omega, \beta^{-1}\right)$ into $L^{2}(\Omega)^{3}$ is not compact (see, for instance, [2]), but that those of $X_{N}\left(\Omega, \beta^{-1}\right)$ and $X_{T}\left(\Omega, \beta^{-1}\right)$ are $($ see $[13])$.

Concerning the traces of vector fields of $H(\operatorname{curl} ; \Omega)$, the following integration by parts formula, or Green's formula, holds

$$
\int_{\Omega} \boldsymbol{u} \cdot \operatorname{curl} \boldsymbol{v} \mathrm{d} \boldsymbol{x}-\int_{\Omega} \operatorname{curl} \boldsymbol{u} \cdot \boldsymbol{v} \mathrm{d} \boldsymbol{x}=\langle\boldsymbol{u} \wedge \boldsymbol{n}, \boldsymbol{n} \wedge(\boldsymbol{v} \wedge \boldsymbol{n})\rangle_{\Gamma},
$$

$$
\forall \boldsymbol{u} \in H(\operatorname{curl} ; \Omega), \forall \boldsymbol{v} \in H(\operatorname{curl} ; \Omega),
$$

where $\boldsymbol{u} \wedge \boldsymbol{n}$ and $\boldsymbol{n} \wedge(\boldsymbol{v} \wedge \boldsymbol{n})$ are respectively the tangential trace of $\boldsymbol{u}$ and the trace of the tangential components of $\boldsymbol{v}$, and $\langle\cdot, \cdot\rangle_{\Gamma}$ stands for the duality product between $H^{-1 / 2}\left(\operatorname{div}_{\Gamma} ; \Gamma\right)$ and its dual $H^{-1 / 2}\left(\operatorname{curl}_{\Gamma} ; \Gamma\right)$, both endowed with their natural quotient norm (see [6,7] or [4] for a summary). In addition, the trace mapping $\boldsymbol{u} \mapsto \boldsymbol{u} \wedge \boldsymbol{n}_{\left.\right|_{\Gamma}}$ $\left(\right.$ resp. $\boldsymbol{v} \mapsto \boldsymbol{n} \wedge(\boldsymbol{v} \wedge \boldsymbol{n})_{\left.\right|_{\Gamma}}$ is onto from $H(\operatorname{curl} ; \Omega)$ to $H^{-1 / 2}\left(\operatorname{div}_{\Gamma} ; \Gamma\right)\left(\operatorname{resp} . H^{-1 / 2}\left(\operatorname{curl}_{\Gamma} ; \Gamma\right)\right)$.

When the domain $\Omega$ is not simply connected, we introduce the kernel

$$
\mathcal{H}\left(m, \beta^{-1}\right)=\left\{\boldsymbol{v} \in X_{T}\left(\Omega, \beta^{-1}\right) \mid \operatorname{div}\left(\beta^{-1} \boldsymbol{v}\right)=0 \text { in } \Omega \text { and } \operatorname{curl} \boldsymbol{v}=\mathbf{0} \text { in } \Omega\right\},
$$

and assume there exists a set of $m$ connected open surfaces $\Sigma_{j}, 1 \leq j \leq m, m$ being the genus of $\partial \Omega$, called "cuts", which allow one to reduce $\Omega$ to a simply-connected domain denoted $\dot{\Omega}=\Omega \backslash \cup_{j=1, \ldots, m} \Sigma_{j}$. 
Similarly, when the boundary $\Gamma$ is not connected, we denote by $\Gamma_{i}, 0 \leq i \leq p$, its (maximal) connected components, $\Gamma_{0}$ being the boundary of the only unbounded connected component of $\mathbb{R}^{3} \backslash \bar{\Omega}$, and we introduce the space

$$
\mathcal{H}\left(e, \beta^{-1}\right)=\left\{\boldsymbol{v} \in X_{N}\left(\Omega, \beta^{-1}\right) \mid \operatorname{div}\left(\beta^{-1} \boldsymbol{v}\right)=0 \text { in } \Omega \text { and } \operatorname{curl} \boldsymbol{v}=\mathbf{0} \text { in } \Omega\right\} .
$$

The proofs of Propositions 3.14 and 3.18 in [2] can be easily adapted to characterize the spaces $\mathcal{H}\left(m, \beta^{-1}\right)$ and $\mathcal{H}\left(e, \beta^{-1}\right)$ as follows.

Proposition 2.1. The dimension of the space $\mathcal{H}\left(m, \beta^{-1}\right)$ is equal to $m$. It is spanned by the functions $\widetilde{\nabla q_{j}^{T}}$, $1 \leq j \leq m$, where each $q_{j}^{T}$ is the solution in $H^{1}(\dot{\Omega})$, unique up to an additive constant, to the problem

$$
\left\{\begin{array}{l}
-\operatorname{div}\left(\beta^{-1} \nabla q_{j}^{T}\right)=0 \text { in } \dot{\Omega}, \\
\partial_{\boldsymbol{n}} q_{j}^{T}=0 \text { on } \Gamma, \\
{\left[q_{j}^{T}\right]_{\Sigma_{k}}=\text { constant and }\left[\beta^{-1} \partial_{\boldsymbol{n}} q_{j}^{T}\right]_{\Sigma_{k}}=0,1 \leq k \leq m,} \\
\left\langle\beta^{-1} \partial_{\boldsymbol{n}} q_{j}^{T}, 1\right\rangle_{\Sigma_{k}}=\delta_{j k}, 1 \leq k \leq m .
\end{array}\right.
$$

Above, given $g \in L^{2}(\dot{\Omega}), \widetilde{g}$ denotes its canonical extension to $L^{2}(\Omega)$.

Proposition 2.2. The dimension of the space $\mathcal{H}\left(e, \beta^{-1}\right)$ is equal to $p$. It is spanned by the functions $\nabla q_{i}^{N}$, $1 \leq i \leq p$, where each $q_{i}^{N}$ is the unique solution in $H^{1}(\Omega)$ to the problem

$$
\left\{\begin{array}{l}
-\operatorname{div}\left(\beta^{-1} \nabla q_{i}^{N}\right)=0 \text { in } \Omega, \\
\left.q_{i}^{N}\right|_{\Gamma_{0}}=0 \text { and }\left.q_{i}^{N}\right|_{\Gamma_{\ell}}=\text { constant }, 1 \leq \ell \leq p, \\
\left\langle\beta^{-1} \partial_{\boldsymbol{n}} q_{i}^{N}, 1\right\rangle_{\Gamma_{0}}=-1 \text { and }\left\langle\beta^{-1} \partial_{\boldsymbol{n}} q_{i}^{N}, 1\right\rangle_{\Gamma_{\ell}}=\delta_{i \ell}, 1 \leq \ell \leq p .
\end{array}\right.
$$

Let $P_{m}$ (resp. $\left.P_{e}\right)$ denote the orthogonal projection from $X_{T}\left(\Omega, \beta^{-1}\right)$ onto $\mathcal{H}\left(m, \beta^{-1}\right)$ (resp. from $X_{N}\left(\Omega, \beta^{-1}\right)$ onto $\mathcal{H}\left(e, \beta^{-1}\right)$ ), with respect to the weighted $L^{2}(\Omega)$-inner product

$$
(\boldsymbol{v}, \boldsymbol{w})_{\beta^{-1}}=\int_{\Omega} \beta^{-1} \boldsymbol{v} \cdot \boldsymbol{w} \mathrm{d} \boldsymbol{x} .
$$

As a consequence of the last two propositions, the projection $P_{m} \boldsymbol{u}$ (resp. $P_{e} \boldsymbol{u}$ ) of a field $\boldsymbol{u}$ satisfying $\operatorname{div}\left(\beta^{-1} \boldsymbol{u}\right)=0$ in $\Omega$ is completely characterized by the set of scalars $\left\langle\beta^{-1} \boldsymbol{u} \cdot \boldsymbol{n}, 1\right\rangle_{\Sigma_{j}}, 1 \leq j \leq m,\left(\operatorname{resp} .\left\langle\beta^{-1} \boldsymbol{u} \cdot \boldsymbol{n}, 1\right\rangle_{\Gamma_{i}}, 1 \leq i \leq p\right)$.

Finally, we recall the following relationship

$$
\operatorname{div}_{\tau}\left(\boldsymbol{v} \wedge \boldsymbol{n}_{\mid \Gamma}\right)=(\operatorname{curl} \boldsymbol{v}) \cdot \boldsymbol{n}_{\mid \Gamma}
$$

which is valid for any (sufficiently smooth) vector field $\boldsymbol{v}$ defined in $\Omega$.

3. An auxiliary problem. In this section, we study existence and uniqueness of a solution to the following auxiliary problem, derived from system (1.1) and constitutive relations (1.2): given $\boldsymbol{f} \in H_{0}\left(\operatorname{div} \beta^{-1} 0 ; \Omega\right)$, find $\boldsymbol{u} \in X_{N}\left(\Omega, \beta^{-1}\right)$ satisfying

$$
\boldsymbol{u}+\beta \operatorname{curl} \boldsymbol{u}=\boldsymbol{f} \text { in } \Omega .
$$

We take inspiration from the work of Boulmezaoud et al. [5] on the existence and uniqueness of Beltrami (or force-free) fields in a bounded domain, with the preliminary consideration of a curl-div system.

3.1. Preliminary study of a curl-div system. We now extend some of the results previously obtained in [5] to a more general context. We introduce the following problem: given $\boldsymbol{j} \in H\left(\operatorname{div} \beta^{-1} 0 ; \Omega\right) \cap \mathcal{H}\left(e, \beta^{-1}\right)^{\perp}$, find $\boldsymbol{u} \in X_{T}\left(\Omega, \beta^{-1}\right)$ such that

$$
\beta \operatorname{curl} \boldsymbol{u}=\boldsymbol{j}, \operatorname{div}\left(\beta^{-1} \boldsymbol{u}\right)=0, \text { and } P_{m} \boldsymbol{u}=\mathbf{0} .
$$

This problem was studied in Lemma 5 of $[5]$ in the case $\beta=1$ and for a smooth domain. We adapt it to our setting (that is, to the case of a non constant parameter $\beta$ and a Lipschitz domain) as follows.

Lemma 3.1. A field $\boldsymbol{u} \in X_{T}\left(\Omega, \beta^{-1}\right)$ is solution to (3.2) if and only if it solves the variational problem

$$
\begin{aligned}
(\beta \operatorname{curl} \boldsymbol{u}, \operatorname{curl} \boldsymbol{v})_{L^{2}(\Omega)^{3}}+\left(\operatorname{div}\left(\beta^{-1} \boldsymbol{u}\right), \operatorname{div}\left(\beta^{-1} \boldsymbol{v}\right)\right)_{L^{2}(\Omega)^{3}} \\
+\left(P_{m} \boldsymbol{u}, P_{m} \boldsymbol{v}\right)_{\beta^{-1}}=(\boldsymbol{j}, \operatorname{curl} \boldsymbol{v})_{L^{2}(\Omega)^{3}}, \forall \boldsymbol{v} \in X_{T}\left(\Omega, \beta^{-1}\right) .
\end{aligned}
$$


Moreover, this variational problem admits a unique solution.

Proof. The existence and uniqueness of a solution to (3.3) simply follows from the Lax-Milgram lemma, the lefthand side of (3.3) defining a coercive and continuous bilinear form on $X_{T}\left(\Omega, \beta^{-1}\right) \times X_{T}\left(\Omega, \beta^{-1}\right)$ as a consequence of Corollary 3.16 in [2]. It remains to show the equivalence between (3.2) and (3.3). As problem (3.2) obviously implies problem (3.3), let us concentrate on proving the converse statement by following the steps given in [5]. Let $\boldsymbol{u} \in X_{T}\left(\Omega, \beta^{-1}\right)$ be a solution to (3.3). First, taking $\boldsymbol{v}=P_{m} \boldsymbol{u}$ as a test function, we find that $\left(P_{m} \boldsymbol{u}, P_{m} \boldsymbol{u}\right)_{\beta^{-1}}=0$, hence $P_{m} \boldsymbol{u}=\mathbf{0}$ almost everywhere in $\Omega$. Next, consider $\varphi \in H^{1}(\Omega) / \mathbb{R}$, solution to the variational problem

$$
\int_{\Omega} \beta^{-1} \nabla \varphi \cdot \nabla \psi \mathrm{d} \boldsymbol{x}=-\int_{\Omega} \operatorname{div}\left(\beta^{-1} \boldsymbol{u}\right) \psi \mathrm{d} \boldsymbol{x}, \forall \psi \in H^{1}(\Omega) .
$$

Such a solution exists since the right-hand side is orthogonal to 1 by the Green formula, namely

$$
\int_{\Omega} \operatorname{div}\left(\beta^{-1} \boldsymbol{u}\right) \mathrm{d} \boldsymbol{x}=\left\langle\beta^{-1} \boldsymbol{u} \cdot \boldsymbol{n}, 1\right\rangle_{\Gamma}=0 .
$$

Furthermore, it is governed by the Neumann problem

$$
\operatorname{div}\left(\beta^{-1} \nabla \varphi\right)=\operatorname{div}\left(\beta^{-1} \boldsymbol{u}\right) \text { in } \Omega, \beta^{-1} \nabla \varphi \cdot \boldsymbol{n}=0 \text { on } \Gamma .
$$

Taking $\boldsymbol{v}=\boldsymbol{\nabla} \varphi$ in (3.3), we deduce that

$$
\left(\operatorname{div}\left(\beta^{-1} \boldsymbol{u}\right), \operatorname{div}\left(\beta^{-1} \nabla \varphi\right)\right)_{L^{2}(\Omega)^{3}}=0,
$$

and therefore $\operatorname{div}\left(\beta^{-1} \boldsymbol{u}\right)=0$ almost everywhere in $\Omega$. Finally, let us set $\boldsymbol{w}=\beta \operatorname{curl} \boldsymbol{u}-\boldsymbol{j}$. Then, using (3.3) and the first two steps, we have

$$
(\boldsymbol{w}, \operatorname{curl} \boldsymbol{v})_{L^{2}(\Omega)^{3}}=0, \forall \boldsymbol{v} \in X_{T}\left(\Omega, \beta^{-1}\right) .
$$

By virtue of Lemma 3.5 in [2], this implies that

$$
(\boldsymbol{w}, \operatorname{curl} \boldsymbol{\Phi})_{L^{2}(\Omega)^{3}}=0, \forall \boldsymbol{\Phi} \in H^{1}(\Omega)^{3} .
$$

Taking $\boldsymbol{\Phi}$ in $\mathscr{D}(\Omega)^{3}$, we then find that $\operatorname{curl} \boldsymbol{w}=\mathbf{0}$ in $\Omega$, which guarantees that $\boldsymbol{w}$ is in $H(\operatorname{curl} ; \Omega)$. Coming back to (3.4) and applying the Green formula, we obtain

$$
\langle\boldsymbol{w} \wedge \boldsymbol{n}, \boldsymbol{\Phi}\rangle_{\Gamma}=0, \forall \boldsymbol{\Phi} \in H^{1}(\Omega)^{3} .
$$

In other words, the field $\boldsymbol{w}$ belongs to $H_{0}(\operatorname{curl} ; \Omega)$. Since $\operatorname{div}\left(\beta^{-1} \boldsymbol{w}\right)=0$ in $\Omega$, we infer that $\boldsymbol{w}$ belongs to $\mathcal{H}\left(e, \beta^{-1}\right)$. Finally, seeing that $P_{e} \boldsymbol{w}=\mathbf{0}$ since

$$
\left\langle\beta^{-1} \boldsymbol{w} \cdot \boldsymbol{n}, 1\right\rangle_{\Gamma_{i}}=\langle\operatorname{curl} \boldsymbol{u} \cdot \boldsymbol{n}, 1\rangle_{\Gamma_{i}}-\left\langle\beta^{-1} \boldsymbol{j} \cdot \boldsymbol{n}, 1\right\rangle_{\Gamma_{i}}=0, \forall i \in\{1, \ldots, p\},
$$

by (2.2) and the assumption on $\boldsymbol{j}$, we deduce, using Proposition 2.2, that $\boldsymbol{w}=\mathbf{0}$ in $\Omega$.

As in [5], we now introduce the (bounded linear) operator from $H\left(\operatorname{div} \beta^{-1} 0 ; \Omega\right) \cap \mathcal{H}\left(e, \beta^{-1}\right)^{\perp}$ into itself

$$
K_{\beta}: \boldsymbol{j} \mapsto \boldsymbol{u},
$$

where $\boldsymbol{u} \in X_{T}\left(\Omega, \beta^{-1}\right)$ is the unique solution to problem (3.2). Since $X_{T}\left(\Omega, \beta^{-1}\right) \cap H\left(\operatorname{div} \beta^{-1} 0 ; \Omega\right)$ is compactly embedded into $H\left(\operatorname{div} \beta^{-1} 0 ; \Omega\right) \cap \mathcal{H}\left(e, \beta^{-1}\right)^{\perp}$, the operator $K_{\beta}$ is clearly compact. Accordingly, the operator $I d+K_{\beta}$ is a Fredholm operator of index zero from $H\left(\operatorname{div} \beta^{-1} 0 ; \Omega\right) \cap \mathcal{H}\left(e, \beta^{-1}\right)^{\perp}$ into itself. Therefore, it is an isomorphism if and only if its kernel is reduced to the null subspace, and the same goes for the operator $I d+K_{\beta}{ }^{*}$, where $K_{\beta}{ }^{*}$ denotes the adjoint of the operator $K_{\beta}$, since $\operatorname{dim} \operatorname{ker}\left(I d+K_{\beta}\right)=\operatorname{dim} \operatorname{ker}\left(I d+K_{\beta}{ }^{*}\right)$ according the Riesz-Fredholm theory.

In order to identify the adjoint of $K_{\beta}$, we need to establish a Weyl-Helmholtz type decomposition for the elements of $H\left(\operatorname{div} \beta^{-1} 0 ; \Omega\right) \cap \mathcal{H}\left(e, \beta^{-1}\right)^{\perp}$. We start by proving the following result.

Lemma 3.2. Let $\boldsymbol{u}$ in $L^{2}(\Omega)^{3}$ be a divergence-free field such that $\boldsymbol{u} \cdot \boldsymbol{n}=0$ on $\Gamma$ and $P_{m} \boldsymbol{u}=\mathbf{0}$. Then, there exists one, and only one, field $\phi$ in $X_{N}\left(\Omega, \beta^{-1}\right)$ such that

$$
\boldsymbol{u}=\operatorname{curl} \boldsymbol{\phi} \text { in } \Omega, \operatorname{div}\left(\beta^{-1} \boldsymbol{\phi}\right)=0 \text { in } \Omega \text {, and } P_{e} \boldsymbol{\phi}=\mathbf{0} .
$$


Proof. By Theorem 3.17 of [2], there exists $\boldsymbol{\psi} \in X_{N}(\Omega, 1)$ such that $\boldsymbol{u}=\operatorname{curl} \boldsymbol{\psi}$ in $\Omega$ and fulfilling the statement of the lemma with $\beta=1$. Since $\boldsymbol{\psi}$ doesn't verify $\operatorname{div}\left(\beta^{-1} \boldsymbol{\psi}\right)=0$ in $\Omega$, we consider $\chi \in H_{0}^{1}(\Omega)$ such that

$$
(\boldsymbol{\nabla} \chi, \boldsymbol{\nabla} v)_{\beta^{-1}}=(\boldsymbol{\psi}, \boldsymbol{\nabla} v)_{\beta^{-1}}, \forall v \in H_{0}^{1}(\Omega) .
$$

This field satisfies

$$
\operatorname{div}\left(\beta^{-1} \nabla \chi\right)=\operatorname{div}\left(\beta^{-1} \boldsymbol{\psi}\right) \text { in } \Omega
$$

and the boundary condition

$$
\beta^{-1} \nabla \chi \wedge \boldsymbol{n}=0 \text { on } \Gamma .
$$

Consequently, the function $\boldsymbol{\phi}=\boldsymbol{\psi}-\boldsymbol{\nabla} \chi-\sum_{i=1}^{p}\left\langle\beta^{-1}(\boldsymbol{\psi}-\boldsymbol{\nabla} \chi) \cdot \boldsymbol{n}, 1\right\rangle_{\Gamma_{i}} \boldsymbol{\nabla} q_{i}^{N}$ meets the requested properties. To conclude that the field $\boldsymbol{\phi}$ is unique, we make the following observation: if $\boldsymbol{u}=\mathbf{0}, \boldsymbol{\phi}$ belongs to $\mathcal{H}\left(e, \beta^{-1}\right)$ and is such that $P_{e} \boldsymbol{\phi}=\mathbf{0}$, so $\boldsymbol{\phi}$ is zero. $\square$

We next give a decomposition for any vector field in $H\left(\operatorname{div} \beta^{-1} 0 ; \Omega\right) \cap \mathcal{H}\left(e, \beta^{-1}\right)^{\perp}$.

Lemma 3.3. Let $\boldsymbol{j} \in H\left(\operatorname{div} \beta^{-1} 0 ; \Omega\right) \cap \mathcal{H}\left(e, \beta^{-1}\right)^{\perp}$ be given. Then, there exist $s \in H^{1}(\Omega) / \mathbb{R}$, coefficients $c_{j}$, $1 \leq j \leq m$, and $\phi \in X_{N}\left(\Omega, \beta^{-1}\right)$ such that

$$
\boldsymbol{j}=\boldsymbol{\nabla} s+\sum_{j=1}^{m} c_{j} \boldsymbol{\nabla} q_{j}^{T}+\beta \operatorname{curl} \boldsymbol{\phi}, \text { with } \operatorname{div}\left(\beta^{-1} \boldsymbol{\phi}\right)=0 \text { in } \Omega \text { and } P_{e} \boldsymbol{\phi}=\mathbf{0} .
$$

The fields $s$ and $\phi$, and the coefficients $\left(c_{j}\right)_{1 \leq j \leq m}$ are unique.

Proof. Consider the function $s \in H^{1}(\Omega) / \mathbb{R}$, solution to the Neumann problem

$$
\operatorname{div}\left(\beta^{-1} \nabla s\right)=0 \text { in } \Omega, \beta^{-1} \nabla s \cdot \boldsymbol{n}=\beta^{-1} \boldsymbol{j} \cdot \boldsymbol{n} \text { on } \Gamma,
$$

which exists since

$$
\left\langle\beta^{-1} \boldsymbol{j} \cdot \boldsymbol{n}, 1\right\rangle_{\Gamma}=\int_{\Omega} \operatorname{div}\left(\beta^{-1} \boldsymbol{j}\right) \mathrm{d} \boldsymbol{x}=0 .
$$

The coefficients $c_{j}, 1 \leq j \leq m$, are then given by

$$
c_{j}=\left\langle\beta^{-1}(\boldsymbol{j}-\nabla s) \cdot \boldsymbol{n}, 1\right\rangle_{\Sigma_{j}} .
$$

As the field $\boldsymbol{u}=\beta^{-1}\left(\boldsymbol{j}-\nabla s-\sum_{j=1}^{m} c_{j} \nabla q_{j}^{T}\right)$ satisfies the hypotheses of Lemma 3.2, we deduce the existence of the vector field $\phi$.

By construction, it is easily seen that the fields $s, \phi$, and the coefficients $\left(c_{j}\right)_{1 \leq j \leq m}$ are unique.

We may now introduce the operator $K^{\star}$ from $H\left(\operatorname{div} \beta^{-1} 0 ; \Omega\right) \cap \mathcal{H}\left(e, \beta^{-1}\right)^{\perp}$ into itself such that

$$
K^{\star}: j \mapsto \phi,
$$

where $\phi$ is the field appearing in Lemma 3.3 (and which depends continuously on $\boldsymbol{j}$ ). It turns out that this operator is the adjoint of $K_{\beta}$ (i.e., $K^{\star}=K_{\beta}{ }^{*}$ ).

Lemma 3.4. The operator $K^{\star}$ is the adjoint of $K_{\beta}$ when $H\left(\operatorname{div} \beta^{-1} 0 ; \Omega\right) \cap \mathcal{H}\left(e, \beta^{-1}\right)^{\perp}$ is equipped with the inner product (2.1).

Proof. Using Lemma 3.3, we can write

$$
\boldsymbol{v}=\boldsymbol{\nabla} s+\sum_{j=1}^{m} c_{j} \boldsymbol{\nabla} q_{j}^{T}+\beta \operatorname{curl} K^{\star} \boldsymbol{v}, \forall \boldsymbol{v} \in H\left(\operatorname{div} \beta^{-1} 0 ; \Omega\right) \cap \mathcal{H}\left(e, \beta^{-1}\right)^{\perp} .
$$

Hence, for any $\boldsymbol{u}$ in $H\left(\operatorname{div} \beta^{-1} 0 ; \Omega\right) \cap \mathcal{H}\left(e, \beta^{-1}\right)^{\perp}$, we have

$$
\left(K_{\beta} \boldsymbol{u}, \boldsymbol{v}\right)_{\beta^{-1}}=\int_{\Omega} K_{\beta} \boldsymbol{u} \cdot\left(\beta^{-1} \nabla s+\beta^{-1} \sum_{j=1}^{m} c_{j} \nabla q_{j}^{T}+\operatorname{curl} K^{\star} \boldsymbol{v}\right) \mathrm{d} \boldsymbol{x} .
$$


Integrating by parts, we see that

$$
\left(K_{\beta} \boldsymbol{u}, \nabla s\right)_{\beta^{-1}}=-\int_{\Omega} \operatorname{div}\left(\beta^{-1} K_{\beta} \boldsymbol{u}\right) s \mathrm{~d} \boldsymbol{x}+\left\langle\beta^{-1} K_{\beta} \boldsymbol{u} \cdot \boldsymbol{n}, s\right\rangle_{\Gamma}=0,
$$

since $\operatorname{div}\left(\beta^{-1} K_{\beta} \boldsymbol{u}\right)=0$ in $\Omega$ and $K_{\beta} \boldsymbol{u}$ belongs to $X_{T}\left(\Omega, \beta^{-1}\right)$. On the other hand, we find that

$$
\left(K_{\beta} \boldsymbol{u}, \nabla q_{j}^{T}\right)_{\beta^{-1}}=\left(P_{m}\left(K_{\beta} \boldsymbol{u}\right), \nabla q_{j}^{T}\right)_{\beta^{-1}}=0
$$

as $P_{m}\left(K_{\beta} \boldsymbol{u}\right)=0$. Consequently, one has

$$
\left(K_{\beta} \boldsymbol{u}, \boldsymbol{v}\right)_{\beta^{-1}}=\int_{\Omega} K_{\beta} \boldsymbol{u} \cdot \operatorname{curl} K^{\star} \boldsymbol{v} \mathrm{d} \boldsymbol{x} .
$$

Applying the Green formula and using the fact that $\left(K^{\star} \boldsymbol{v}\right) \wedge \boldsymbol{n}=\mathbf{0}$ on $\Gamma$, we reach

$$
\left(K_{\beta} \boldsymbol{u}, \boldsymbol{v}\right)_{\beta^{-1}}=\int_{\Omega} \operatorname{curl}\left(K_{\beta} \boldsymbol{u}\right) \cdot K^{\star} \boldsymbol{v} \mathrm{d} \boldsymbol{x},
$$

and, since $\operatorname{curl}\left(K_{\beta} \boldsymbol{u}\right)=\beta^{-1} \boldsymbol{u}$, we have finally proved that

$$
\left(K_{\beta} \boldsymbol{u}, \boldsymbol{v}\right)_{\beta^{-1}}=\left(\boldsymbol{u}, K^{\star} \boldsymbol{v}\right)_{\beta^{-1}}
$$

$$
\forall \boldsymbol{u} \in H\left(\operatorname{div} \beta^{-1} 0 ; \Omega\right) \cap \mathcal{H}\left(e, \beta^{-1}\right)^{\perp}, \forall \boldsymbol{v} \in H\left(\operatorname{div} \beta^{-1} 0 ; \Omega\right) \cap \mathcal{H}\left(e, \beta^{-1}\right)^{\perp}
$$

3.2. The Drude-Born-Fedorov operator. So far, we have not used the fact that $\beta$ is piecewise regular, an assumption which appeared before stating the proportionality assumptions (1.3). From now on, it will be explicitly needed, starting with Theorem 3.5 .

We next consider the mapping

$$
\begin{aligned}
A_{\beta}: & X_{N}\left(\Omega, \beta^{-1}\right) \rightarrow H\left(\operatorname{div} \beta^{-1} ; \Omega\right) \\
& \boldsymbol{u} \mapsto \boldsymbol{u}+\beta \operatorname{curl} \boldsymbol{u},
\end{aligned}
$$

defining the so-called Drude-Born-Fedorov operator, and suppose that $H\left(\operatorname{div} \beta^{-1} ; \Omega\right)$ is equipped with the inner product

$$
(\boldsymbol{u}, \boldsymbol{v})_{H}=(\boldsymbol{u}, \boldsymbol{v})_{\beta^{-1}}+\left(\operatorname{div}\left(\beta^{-1} \boldsymbol{u}\right), \operatorname{div}\left(\beta^{-1} \boldsymbol{v}\right)\right)_{L^{2}(\Omega)},
$$

$$
\forall \boldsymbol{u} \in H\left(\operatorname{div} \beta^{-1} ; \Omega\right), \forall \boldsymbol{v} \in H\left(\operatorname{div} \beta^{-1} ; \Omega\right)
$$

Let us characterize the orthogonal to the range of operator $A_{\beta}$ in $H\left(\operatorname{div} \beta^{-1} ; \Omega\right)$. We first remark that $A_{\beta} \boldsymbol{v}=\boldsymbol{v}$ for any $\boldsymbol{v} \in \mathcal{H}\left(e, \beta^{-1}\right)$, so that $\mathcal{H}\left(e, \beta^{-1}\right) \subset R\left(A_{\beta}\right)$. Also, according to the definition of $P_{e}$ (with respect to the scalar product $\left.(\cdot, \cdot)_{\beta^{-1}}\right)$, one finds that

$$
P_{e} \boldsymbol{w}=\mathbf{0}, \forall \boldsymbol{w} \in R\left(A_{\beta}\right)^{\perp}
$$

THEOREM 3.5. In $H\left(\operatorname{div} \beta^{-1} ; \Omega\right)$, one has

$$
R\left(A_{\beta}\right)^{\perp}=\left\{\boldsymbol{w} \in H\left(\operatorname{div} \beta^{-1} ; \Omega\right) \mid \boldsymbol{w}+\beta \operatorname{curl} \boldsymbol{w}=\mathbf{0}\right\} .
$$

Proof. Let us start with the inclusion $R\left(A_{\beta}\right)^{\perp} \subset\left\{\boldsymbol{w} \in H\left(\operatorname{div} \beta^{-1} ; \Omega\right) \mid \boldsymbol{w}+\beta \operatorname{curl} \boldsymbol{w}=\mathbf{0}\right\}$. Let $\boldsymbol{w} \in R\left(A_{\beta}\right)^{\perp}$; it is such that

$$
0=\left(A_{\beta} \boldsymbol{v}, \boldsymbol{w}\right)_{H}=(\boldsymbol{v}+\beta \operatorname{curl} \boldsymbol{v}, \boldsymbol{w})_{\beta^{-1}}+\left(\operatorname{div}\left(\beta^{-1} \boldsymbol{v}\right), \operatorname{div}\left(\beta^{-1} \boldsymbol{w}\right)\right)_{L^{2}(\Omega)}, \quad, \quad \forall \boldsymbol{v} \in X_{N}\left(\Omega, \beta^{-1}\right)
$$

For any $g \in L^{2}(\Omega)$, let $\varphi \in H_{0}^{1}(\Omega)$ be the unique solution to

$$
\operatorname{div}\left(\beta^{-1} \nabla \varphi\right)-\varphi=g \text { in } \Omega
$$


in the following sense

$$
\int_{\Omega}\left(\beta^{-1} \nabla \varphi \cdot \nabla \chi+\varphi \chi\right) \mathrm{d} \boldsymbol{x}=-\int_{\Omega} g \chi \mathrm{d} \boldsymbol{x}, \forall \chi \in H_{0}^{1}(\Omega),
$$

and consider in (3.8) the test function $\boldsymbol{v}=\boldsymbol{\nabla} \varphi$. This implies that

$$
(\boldsymbol{\nabla} \varphi, \boldsymbol{w})_{\beta^{-1}}+\left(\operatorname{div}\left(\beta^{-1} \boldsymbol{\nabla} \varphi\right), \operatorname{div}\left(\beta^{-1} \boldsymbol{w}\right)\right)_{L^{2}(\Omega)}=0 .
$$

Integrating by parts the first term in the left-hand side, we obtain

$$
0=\left(-\varphi+\operatorname{div}\left(\beta^{-1} \nabla \varphi\right), \operatorname{div}\left(\beta^{-1} \boldsymbol{w}\right)\right)_{L^{2}(\Omega)}=\left(g, \operatorname{div}\left(\beta^{-1} \boldsymbol{w}\right)_{L^{2}(\Omega)}, \forall g \in L^{2}(\Omega) .\right.
$$

In other words, one has

$$
\operatorname{div}\left(\beta^{-1} \boldsymbol{w}\right)=0 \text { in } \Omega,
$$

and we see, by applying the Green formula, that (3.8) reduces to

$$
(\boldsymbol{v}, \boldsymbol{w}+\beta \operatorname{curl} \boldsymbol{w})_{\beta^{-1}}=0, \forall \boldsymbol{v} \in X_{N}\left(\Omega, \beta^{-1}\right) .
$$

Now, for any $\boldsymbol{z}$ in $\mathscr{D}\left(\Omega_{k}\right)^{3}, 1 \leq k \leq K$, let us set $\boldsymbol{v}=\boldsymbol{z}-\boldsymbol{\nabla} \psi$, where $\psi$ belongs to $H_{0}^{1}\left(\Omega_{k}\right)$ and is such that $\operatorname{div}\left(\beta^{-1} \boldsymbol{\nabla} \psi\right)=\operatorname{div}\left(\beta^{-1} \boldsymbol{z}\right)$ in $\Omega_{k}$. By construction, the field $\boldsymbol{v}$ belongs to $X_{N}\left(\Omega, \beta^{-1}\right)$ and plugging this test function in equality $(3.10)$ yields

$$
\boldsymbol{w}+\beta \operatorname{curl} \boldsymbol{w}=\mathbf{0} \text { in } \Omega_{k} .
$$

Hence, the field $\boldsymbol{w}$ belongs to $H\left(\operatorname{curl}, \Omega_{k}\right)$ and we can apply the Green formula on each subdomain $\Omega_{k}$ to get

$$
\sum_{k=1}^{K}\langle\boldsymbol{w} \wedge \boldsymbol{n}, \boldsymbol{n} \wedge(\boldsymbol{v} \wedge \boldsymbol{n})\rangle_{\partial \Omega_{k}}=0, \forall \boldsymbol{v} \in X_{N}\left(\Omega, \beta^{-1}\right) .
$$

Hence, the tangential components of $\boldsymbol{w}$ are continuous across interfaces between subdomains. This implies that $\boldsymbol{w}$ belongs to $H(\operatorname{curl} ; \Omega)$ and satisfies

$$
\boldsymbol{w}+\beta \operatorname{curl} \boldsymbol{w}=\mathbf{0} \text { in } \Omega .
$$

The converse inclusion follows directly from using the Green formula and remarking that the identity $\boldsymbol{w}+$ $\beta \operatorname{curl} \boldsymbol{w}=\mathbf{0}$ implies that $\operatorname{div}\left(\beta^{-1} \boldsymbol{w}\right)=0$. $\square$

Lemma 3.6. Assume that

$$
\operatorname{ker}\left(I d+K_{\beta}\right)=\{\mathbf{0}\}
$$

Then, a vector field $\boldsymbol{w}$ belongs to $R\left(A_{\beta}\right)^{\perp}$ if and only if there exists $g$ in $H_{0}^{-1 / 2}(\Gamma)=\left\{g \in H^{-1 / 2}(\Gamma) \mid\langle g, 1\rangle_{\Gamma}=0\right\}$ such that

$$
\boldsymbol{w}=\boldsymbol{w}_{0}+\nabla \varphi_{g}
$$

where $\boldsymbol{w}_{0}=-K_{\beta}\left(I d+K_{\beta}\right)^{-1} \nabla \varphi_{g}$ and $\varphi_{g} \in H^{1}(\Omega) / \mathbb{R}$ is the variational solution to

$$
\begin{aligned}
\operatorname{div}\left(\beta^{-1} \nabla \varphi_{g}\right) & =0 \text { in } \Omega, \\
\beta^{-1} \nabla \varphi_{g} \cdot \boldsymbol{n} & =g \text { on } \Gamma,
\end{aligned}
$$

in the following sense

$$
\int_{\Omega} \beta^{-1} \nabla \varphi_{g} \cdot \nabla \chi \mathrm{d} \boldsymbol{x}=\langle g, \chi\rangle_{\Gamma}, \forall \chi \in H^{1}(\Omega) / \mathbb{R}
$$

Proof. For any $\boldsymbol{w}$ in $R\left(A_{\beta}\right)^{\perp}$, let us denote its trace on $\Gamma$, which is an element of $H_{0}^{-1 / 2}(\Gamma)$, by $g=\beta^{-1} \boldsymbol{w} \cdot \boldsymbol{n}_{\mid \Gamma}$ and set

$$
\boldsymbol{w}_{0}=\boldsymbol{w}-\nabla \varphi_{g}
$$


We remark that $\boldsymbol{w}_{0}$ belongs to $H_{0}\left(\operatorname{div} \beta^{-1} 0 ; \Omega\right) \cap H(\operatorname{curl} ; \Omega)$. Setting $\boldsymbol{j}=\beta \operatorname{curl} \boldsymbol{w}_{0}$, we see that $\boldsymbol{j} \in H\left(\operatorname{div} \beta^{-1} 0 ; \Omega\right) \cap$ $\mathcal{H}\left(e, \beta^{-1}\right)^{\perp}$ and $K_{\beta} \boldsymbol{j}=\boldsymbol{w}_{0}$, and that

$$
\boldsymbol{j}+K_{\beta} \boldsymbol{j}=\boldsymbol{w}_{0}+\beta \operatorname{curl} \boldsymbol{w}_{0}=-\nabla \varphi_{g}
$$

this last equality following from the identity $\boldsymbol{w}+\beta \operatorname{curl} \boldsymbol{w}=\mathbf{0}$. The operator $I d+K_{\beta}$ being invertible due to condition (3.11), we deduce that

$$
\boldsymbol{j}=-\left(I d+K_{\beta}\right)^{-1} \nabla \varphi_{g}
$$

and therefore

$$
\boldsymbol{w}_{0}=K_{\beta} \boldsymbol{j}=-K_{\beta}\left(I d+K_{\beta}\right)^{-1} \nabla \varphi_{g}
$$

Conversely, for any $g$ in $H_{0}^{-1 / 2}(\Gamma)$, let us set $\boldsymbol{w}_{0}=-K_{\beta}\left(I d+K_{\beta}\right)^{-1} \nabla \varphi_{g}$ and

$$
\boldsymbol{w}=\boldsymbol{w}_{0}+\nabla \varphi_{g}
$$

The above arguments show that $\boldsymbol{w}$ is in $H\left(\operatorname{div} \beta^{-1} ; \Omega\right)$ and satisfies $\boldsymbol{w}+\beta \operatorname{curl} \boldsymbol{w}=\mathbf{0}$, so it belongs to $R\left(A_{\beta}\right)^{\perp}$. $\mathrm{u}$

Corollary 3.7. Assume that condition (3.11) holds. Then, there exist nonzero elements $\boldsymbol{f} \in H_{0}\left(\operatorname{div} \beta^{-1} 0 ; \Omega\right)$ such that $\boldsymbol{f} \notin R\left(A_{\beta}\right)$.

Proof. For any nonzero element $g$ in $H_{0}^{-1 / 2}(\Gamma)$, let us set $\boldsymbol{w}_{0}=-K_{\beta}\left(I d+K_{\beta}\right)^{-1} \boldsymbol{\nabla} \varphi_{g}$ and $\boldsymbol{w}=\boldsymbol{w}_{0}+\boldsymbol{\nabla} \varphi_{g}, \varphi_{g}$ being defined as in Lemma 3.6.

On the one hand, Lemma 3.6 shows that the field $\boldsymbol{w}$ belongs to $R\left(A_{\beta}\right)^{\perp}$. On the other hand, the field $\boldsymbol{w}_{0}$ is in $H_{0}\left(\operatorname{div} \beta^{-1} 0 ; \Omega\right)$ and is nonzero (indeed, if were the case, the identity $\boldsymbol{w}_{0}+\beta \operatorname{curl} \boldsymbol{w}_{0}=-\boldsymbol{\nabla} \varphi_{g}$ would imply that $\varphi_{g}=0$ and hence $g$ would be zero).

Finally, $\boldsymbol{w}_{0}$ cannot be in the range of $A_{\beta}$, because it is not orthogonal to $\boldsymbol{w}$. Indeed, one has

$$
\left(\boldsymbol{w}_{0}, \boldsymbol{w}\right)_{H}=\left(\boldsymbol{w}_{0}, \boldsymbol{w}\right)_{\beta^{-1}}=\left(\boldsymbol{w}_{0}, \boldsymbol{w}_{0}+\nabla \varphi_{g}\right)_{\beta^{-1}}=\left(\boldsymbol{w}_{0}, \boldsymbol{w}_{0}\right)_{\beta^{-1}} \neq 0,
$$

as an integration by parts yields $\left(\boldsymbol{w}_{0}, \boldsymbol{\nabla} \varphi_{g}\right)_{\beta^{-1}}=0$.

Let us now go back to the auxiliary problem (3.1). We are now in a position to formulate a non-invertibility result, which is a direct consequence of the preceding corollary.

COROLlaRY 3.8. Under condition $(3.11)$, the operator $A_{\beta}$ is not an isomorphism from $X_{N}\left(\Omega, \beta^{-1}\right) \cap H_{0}\left(\operatorname{div} \beta^{-1} 0 ; \Omega\right)$ into $H_{0}\left(\operatorname{div} \beta^{-1} 0 ; \Omega\right)$.

This last corollary negates an affirmation which was, erroneously, thought to be proved in the case of a homogeneous material in [8]. The correction of this point is one of the main goals of the present work.

Nevertheless, one can establish the following results.

TheOrem 3.9. Assume that condition (3.11) holds. Then, the range $R\left(A_{\beta}\right)$ is closed in $H\left(\operatorname{div} \beta^{-1} ; \Omega\right)$.

Proof. Let us consider a sequence $\left(\boldsymbol{u}_{n}\right)_{n \in \mathbb{N}}$ in $X_{N}\left(\Omega, \beta^{-1}\right)$ such that

$$
\boldsymbol{f}_{n}=\boldsymbol{u}_{n}+\beta \operatorname{curl} \boldsymbol{u}_{n} \rightarrow \boldsymbol{f} \text { in } H \text { as } n \rightarrow+\infty \text {. }
$$

This equivalently means that

$$
\boldsymbol{f}_{n} \rightarrow \boldsymbol{f} \text { in } L^{2}(\Omega)^{3} \text { and } \operatorname{div}\left(\beta^{-1} \boldsymbol{u}_{n}\right) \rightarrow \operatorname{div}\left(\beta^{-1} \boldsymbol{f}\right) \text { in } L^{2}(\Omega), \text { as } n \rightarrow+\infty \text {. }
$$

Let $\varphi_{n}$ in $H_{c}^{1}(\Omega)=\left\{v \in H^{1}(\Omega) \mid v_{\left.\right|_{\Gamma_{0}}}=0, v_{\left.\right|_{\Gamma_{i}}}=\right.$ constant, $\left.1 \leq i \leq p\right\}$ be the unique variational solution to ${ }^{1}$

$$
\operatorname{div}\left(\beta^{-1} \nabla \varphi_{n}\right)=\operatorname{div}\left(\beta^{-1} \boldsymbol{u}_{n}\right) \text { in } \Omega
$$

According to $(3.12)$, the sequence $\left(\varphi_{n}\right)_{n \in \mathbb{N}}$ converges in $H_{c}^{1}(\Omega)$, namely there exists $\varphi \in H_{c}^{1}(\Omega)$ such that

$$
\varphi_{n} \rightarrow \varphi \text { in } H_{c}^{1}(\Omega) \text { as } n \rightarrow+\infty, \text { with } \operatorname{div}\left(\beta^{-1} \nabla \varphi\right)=\operatorname{div}\left(\beta^{-1} \boldsymbol{f}\right)
$$

We next focus our attention on the divergence-free part of $\boldsymbol{u}_{n}, \forall n \in \mathbb{N}$, by setting

$$
\boldsymbol{\Phi}_{n}=\boldsymbol{u}_{n}-\nabla \varphi_{n}
$$

\footnotetext{
${ }^{1}$ Observe that, $\forall n \in \mathbb{N}, \varphi_{n}$ is such that $\left\langle\beta^{-1} \nabla \varphi_{n} \cdot \boldsymbol{n}, 1\right\rangle_{\Gamma_{i}}=\left\langle\beta^{-1} \boldsymbol{u}_{n} \cdot \boldsymbol{n}, 1\right\rangle_{\Gamma_{i}}, 1 \leq i \leq p$.
} 
The sequence $\left(\boldsymbol{\Phi}_{n}\right)_{n \in \mathbb{N}}$ then satisfies

$$
\begin{aligned}
& \operatorname{div}\left(\beta^{-1} \boldsymbol{\Phi}_{n}\right)=0 \text { in } \Omega, \boldsymbol{\Phi}_{n} \wedge \boldsymbol{n}=\mathbf{0} \text { on } \Gamma, P_{e} \boldsymbol{\Phi}_{n}=\mathbf{0}, \forall n \in \mathbb{N}, \\
& \tilde{\boldsymbol{f}}_{n}=\boldsymbol{\Phi}_{n}+\beta \operatorname{curl} \boldsymbol{\Phi}_{n}=\boldsymbol{f}_{n}-\boldsymbol{\nabla} \varphi_{n} \rightarrow \boldsymbol{f}-\boldsymbol{\nabla} \varphi \text { in } L^{2}(\Omega)^{3} \text { as } n \rightarrow+\infty
\end{aligned}
$$

Consider now $\boldsymbol{j}_{n}=\beta \operatorname{curl} \boldsymbol{\Phi}_{n}, \forall n \in \mathbb{N}$. By construction (see (3.5) and (3.6)), we have

$$
\boldsymbol{\Phi}_{n}=K_{\beta}^{*} \boldsymbol{j}_{n}
$$

and consequently

$$
\boldsymbol{j}_{n}+K_{\beta}{ }^{*} \boldsymbol{j}_{n}=\boldsymbol{\Phi}_{n}+\beta \operatorname{curl} \boldsymbol{\Phi}_{n}=\tilde{\boldsymbol{f}}_{n} \rightarrow \boldsymbol{f}-\boldsymbol{\nabla} \varphi \text { in } H\left(\operatorname{div} \beta^{-1} ; \Omega\right) \text { as } n \rightarrow+\infty .
$$

Since the operator $I d+K_{\beta}{ }^{*}$ is bounded from $H\left(\operatorname{div} \beta^{-1} ; \Omega\right)$ into itself and invertible, its inverse is also bounded, which implies that

$$
\boldsymbol{j}_{n}=\left(I d+K_{\beta}^{*}\right)^{-1} \tilde{\boldsymbol{f}}_{n} \rightarrow\left(I d+K_{\beta}^{*}\right)^{-1}(\boldsymbol{f}-\nabla \varphi) \text { in } H\left(\operatorname{div} \beta^{-1} ; \Omega\right) \text { as } n \rightarrow+\infty \text {. }
$$

As $K_{\beta}{ }^{*}$ is bounded, we deduce in turn that

$$
\boldsymbol{\Phi}_{n}=K_{\beta}{ }^{*} \boldsymbol{j}_{n} \rightarrow K_{\beta}{ }^{*}\left(I d+K_{\beta}{ }^{*}\right)^{-1}(\boldsymbol{f}-\nabla \varphi)=\boldsymbol{\Phi} \text { in } H\left(\operatorname{div} \beta^{-1} ; \Omega\right) \text { as } n \rightarrow+\infty
$$

This last property implies that

$$
\boldsymbol{u}_{n}=\boldsymbol{\Phi}_{n}+\nabla \varphi_{n} \rightarrow \boldsymbol{\Phi}+\nabla \varphi=\boldsymbol{u} \text { in } L^{2}(\Omega)^{3} \text { as } n \rightarrow+\infty
$$

as well as $\operatorname{div}\left(\beta^{-1} \boldsymbol{u}\right)=\operatorname{div}\left(\beta^{-1} \boldsymbol{f}\right)$ in $L^{2}(\Omega)$. Moreover, we have

$$
\operatorname{curl} \boldsymbol{u}_{n}=\operatorname{curl} \Phi_{n}=\beta^{-1}\left(\tilde{\boldsymbol{f}}_{n}-\boldsymbol{\Phi}_{n}\right) \rightarrow \beta^{-1}(\boldsymbol{f}-\nabla \varphi-\boldsymbol{\Phi}) \text { in } L^{2}(\Omega)^{3},
$$

so that $\boldsymbol{u}_{n}$ tends to $\boldsymbol{u}$ in $X_{N}\left(\Omega, \beta^{-1}\right)$ as $n$ tends to infinity, with $\operatorname{curl} \boldsymbol{u}=\beta^{-1}(\boldsymbol{f}-\boldsymbol{\nabla} \varphi-\boldsymbol{\Phi})$. This finally leads to

$$
\boldsymbol{u}+\beta \operatorname{curl} \boldsymbol{u}=\boldsymbol{\Phi}+\boldsymbol{\nabla} \varphi+\boldsymbol{f}-\nabla \varphi-\boldsymbol{\Phi}=\boldsymbol{f}
$$

and shows that $\boldsymbol{f}$ indeed belongs to $R\left(A_{\beta}\right)$.

Corollary 3.10. Under condition (3.11), the Drude-Born-Fedorov operator $A_{\beta}$ is an isomorphism from $X_{N}\left(\Omega, \beta^{-1}\right)$ into $R\left(A_{\beta}\right)$.

REMARK 3.1. For a homogeneous isotropic chiral material, that is when the value of the chiral admittance $\beta$ is constant over the whole domain $\Omega$, condition (3.11) means that $\beta$ does not belong to a discrete set of real numbers related to the spectrum of the curl operator (see [5] and [14] for details). Here, as the admittance is not necessarily constant, this condition only expresses that -1 is not an eigenvalue of the operator $K_{\beta}$. We may conjecture that it is however generic, in the sense that if it is satisfied for a given function $\beta_{0}$ then, given a small perturbation $\beta^{\prime}$ of $\beta_{0},-1$ is not an eigenvalue of the operator $K_{\beta^{\prime}}$ either.

We conclude this section with a second decomposition for the elements of $R\left(A_{\beta}\right)^{\perp}$, which is needed in the remainder of the paper.

LEMma 3.11. Assume condition (3.11) holds. Then, a field $\boldsymbol{w}$ belongs to $R\left(A_{\beta}\right)^{\perp}$ if and only if there exists $\boldsymbol{h}$ in $H^{-1 / 2}\left(\operatorname{div}_{\Gamma} ; \Gamma\right)$ such that

$$
\boldsymbol{w}=\boldsymbol{w}_{0}+\boldsymbol{w}_{h}
$$

with $\boldsymbol{w}_{0}=-K_{\beta}{ }^{*}\left(I d+K_{\beta}{ }^{*}\right)^{-1}\left(\boldsymbol{w}_{h}+\beta \operatorname{curl} \boldsymbol{w}_{h}\right)$ and $\boldsymbol{w}_{h} \in H\left(\operatorname{div} \beta^{-1} 0, \Omega\right) \cap H(\mathbf{c u r l} ; \Omega)$ is the variational solution to

$$
\begin{aligned}
& \beta \operatorname{curl}\left(\beta \operatorname{curl} \boldsymbol{w}_{h}\right)=\mathbf{0} \text { in } \Omega, \\
& \boldsymbol{w}_{h} \wedge \boldsymbol{n}=\boldsymbol{h} \text { on } \Gamma \\
& P_{e} \boldsymbol{w}_{h}=P_{e} \boldsymbol{w}
\end{aligned}
$$

in the sense described below.

Nota bene. By construction, one has that $\boldsymbol{w} \wedge \boldsymbol{n}=\boldsymbol{h}$ on $\Gamma$. As a consequence, the tangential trace mapping is surjective from $R\left(A_{\beta}\right)^{\perp}$ to $H^{-1 / 2}\left(\operatorname{div}_{\Gamma} ; \Gamma\right)$. 
Proof. Let $\boldsymbol{w} \in R\left(A_{\beta}\right)^{\perp}$. Then, one has $\operatorname{curl} \boldsymbol{w}=-\beta^{-1} \boldsymbol{w}$ belongs to $L^{2}(\Omega)^{3}$ and therefore $\boldsymbol{w}$ belongs to $H(\operatorname{curl} ; \Omega)$ and $\boldsymbol{h}=\boldsymbol{w} \wedge \boldsymbol{n}$ belongs to $H^{-1 / 2}\left(\operatorname{div}_{\Gamma} ; \Gamma\right)$. Let us now fix an element $\hat{\boldsymbol{w}}$ in $H(\operatorname{curl} ; \Omega)$ such that

$$
\hat{\boldsymbol{w}} \wedge \boldsymbol{n}=\boldsymbol{h} .
$$

Up to subtracting a gradient, we can assume that $\beta^{-1} \hat{\boldsymbol{w}}$ is divergence-free and that it is furthermore orthogonal to $\mathcal{H}\left(e, \beta^{-1}\right)$. For that, consider the unique solution $\hat{\boldsymbol{w}}_{h} \in X_{N}\left(\Omega, \beta^{-1}\right)$ satisfying

$$
\begin{aligned}
\left(\beta \operatorname{curl} \hat{\boldsymbol{w}}_{h}, \operatorname{curl} \boldsymbol{v}\right)_{L^{2}(\Omega)^{3}}+\left(\operatorname{div}\left(\beta^{-1} \hat{\boldsymbol{w}}_{h}\right), \operatorname{div}\left(\beta^{-1} \boldsymbol{v}\right)\right)_{L^{2}(\Omega)}+\left(P_{e} \hat{\boldsymbol{w}}_{h}, P_{e} \boldsymbol{v}\right)_{\beta^{-1}}= \\
(\beta \operatorname{curl} \hat{\boldsymbol{w}}, \operatorname{curl} \boldsymbol{v})_{L^{2}(\Omega)^{3}}+\left(P_{e}(\boldsymbol{w}-\hat{\boldsymbol{w}}), P_{e} \boldsymbol{v}\right)_{\beta^{-1}}, \forall \boldsymbol{v} \in X_{N}\left(\Omega, \beta^{-1}\right) .
\end{aligned}
$$

Then, the field

$$
\boldsymbol{w}_{h}=\hat{\boldsymbol{w}}_{h}+\hat{\boldsymbol{w}}
$$

belongs to $H\left(\operatorname{div} \beta^{-1} 0, \Omega\right) \cap H(\operatorname{curl} ; \Omega)$, with $P_{e} \boldsymbol{w}_{h}=P_{e} \boldsymbol{w}$, and is the solution to (3.13). Now we set

$$
\boldsymbol{w}_{0}=\boldsymbol{w}_{h}-\boldsymbol{w}
$$

which is in $X_{N}\left(\Omega, \beta^{-1}\right) \cap H\left(\operatorname{div} \beta^{-1} 0, \Omega\right)$ and such that $P_{e} \boldsymbol{w}_{0}=\mathbf{0}$, and we consider $\boldsymbol{j}^{*}=\beta$ curl $\boldsymbol{w}_{0}$. By $(3.5)$ and (3.6), we have that $\boldsymbol{w}_{0}=K_{\beta}{ }^{*} \boldsymbol{j}^{*}$ and, from (3.16),

$$
\boldsymbol{j}^{*}+K_{\beta}{ }^{*} \boldsymbol{j}^{*}=\beta \operatorname{curl} \boldsymbol{w}_{0}+\boldsymbol{w}_{0}=\beta \operatorname{curl} \boldsymbol{w}_{h}+\boldsymbol{w}_{h} \in H\left(\operatorname{div} \beta^{-1} 0, \Omega\right) .
$$

Since the operator $I d+K_{\beta}{ }^{*}$ is invertible, we deduce that

$$
\boldsymbol{j}^{*}=\left(I d+K_{\beta}{ }^{*}\right)^{-1}\left(\beta \operatorname{curl} \boldsymbol{w}_{h}+\boldsymbol{w}_{h}\right),
$$

hence the decomposition

$$
\boldsymbol{w}=\boldsymbol{w}_{h}-\boldsymbol{w}_{0}=\boldsymbol{w}_{h}-K_{\beta}{ }^{*}\left(I d+K_{\beta}{ }^{*}\right)^{-1}\left(\beta \operatorname{curl} \boldsymbol{w}_{h}+\boldsymbol{w}_{h}\right) .
$$

Conversely, given $\boldsymbol{h}$ in $H^{-1 / 2}\left(\operatorname{div}_{\Gamma} ; \Gamma\right)$, we fix any element $\hat{\boldsymbol{w}}$ in $H\left(\operatorname{div} \beta^{-1} 0, \Omega\right) \cap H(\operatorname{curl} ; \Omega)$ satisfying $P_{e} \hat{\boldsymbol{w}}=\mathbf{0}$ and (3.14) and we consider $\hat{\boldsymbol{w}}_{h}$ the unique solution in $X_{N}\left(\Omega, \beta^{-1}\right)$ to (3.15). The above arguments then show that

$$
\boldsymbol{w}=\boldsymbol{w}_{h}-K_{\beta}^{*}\left(I d+K_{\beta}^{*}\right)^{-1}\left(\beta \operatorname{curl} \boldsymbol{w}_{h}+\boldsymbol{w}_{h}\right)
$$

is an element of $R\left(A_{\beta}\right)^{\perp}$. $\square$

4. Evolution problem. In order to be solved, the original physical problem must be recast in the mathematical framework introduced so far. We thus need to precise the hypotheses on the data.

The a priori assumptions on the initial conditions are that $\boldsymbol{E}_{0} \in X_{N}(\Omega, \beta)$ and $\boldsymbol{H}_{0} \in H(\mathbf{c u r l}, \Omega)$, satisfying $\boldsymbol{H}_{0} \wedge \boldsymbol{n}=\boldsymbol{J}(0)$ on $\Gamma$. Concerning the surface current density $\boldsymbol{J}$, we assume that it is measurable with respect to the time variable $t$. We already know from Section 1 that $\operatorname{div}_{\tau} \boldsymbol{J}(\cdot, t)=0$ on $\Gamma, t \geq 0$, and we furthermore suppose that $\boldsymbol{J}$ is such that there exists a lifting $\boldsymbol{H}_{\boldsymbol{J}}(t), t \geq 0$, with $\boldsymbol{H}_{\boldsymbol{J}}(t) \wedge \boldsymbol{n}=\boldsymbol{J}(t)$ on $\Gamma$. The existence of such a lifting allows us to replace the system (1.1)-(1.2) by an equivalent evolution problem with homogeneous boundary conditions.

As a consequence, the fields $\boldsymbol{E}$ and $\widetilde{\boldsymbol{H}}=\boldsymbol{H}-\boldsymbol{H}_{\boldsymbol{J}}$ satisfy

$$
\left\{\begin{array}{l}
\varepsilon \frac{\partial}{\partial t}(\boldsymbol{E}+\beta \operatorname{curl} \boldsymbol{E})-\operatorname{curl} \widetilde{\boldsymbol{H}}=\operatorname{curl} \boldsymbol{H}_{\boldsymbol{J}} \text { in } \Omega, t>0, \\
\mu \frac{\partial}{\partial t}(\widetilde{\boldsymbol{H}}+\beta \operatorname{curl} \widetilde{\boldsymbol{H}})+\operatorname{curl} \boldsymbol{E}=-\mu \frac{\partial}{\partial t}\left(\boldsymbol{H}_{\boldsymbol{J}}+\beta \operatorname{curl} \boldsymbol{H}_{\boldsymbol{J}}\right) \text { in } \Omega, t>0, \\
\boldsymbol{E} \wedge \boldsymbol{n}=\mathbf{0}, \widetilde{\boldsymbol{H}} \wedge \boldsymbol{n}=\mathbf{0} \text { on } \Gamma, t>0 \\
\boldsymbol{E}(0)=\boldsymbol{E}_{0}, \widetilde{\boldsymbol{H}}(0)=\widetilde{\boldsymbol{H}}_{0}=\boldsymbol{H}_{0}-\boldsymbol{H}_{\boldsymbol{J}}(0) \text { in } \Omega,
\end{array}\right.
$$

which can be written as the following abstract evolution problem: find $\mathscr{E}(t) \in$ $X_{N}\left(\Omega, \beta^{-1}\right) \times X_{N}\left(\Omega, \beta^{-1}\right), t>0$, such that

$$
\left\{\begin{array}{l}
\frac{\mathrm{d}}{\mathrm{d} t}\left(\mathcal{A}_{\beta} \mathscr{E}\right)-\mathcal{C} \mathscr{E}=\mathscr{F}, t>0 \\
\mathscr{E}(0)=\mathscr{E}
\end{array}\right.
$$


by setting

$$
\mathscr{E}=\left(\begin{array}{c}
\boldsymbol{E} \\
\widetilde{\boldsymbol{H}}
\end{array}\right), \mathscr{E}_{0}=\left(\begin{array}{c}
\boldsymbol{E}_{0} \\
\widetilde{\boldsymbol{H}}_{0}
\end{array}\right) \text { and } \mathscr{F}=\left(\begin{array}{c}
\varepsilon^{-1} \operatorname{curl} \boldsymbol{H}_{\boldsymbol{J}} \\
-\frac{\partial}{\partial t}\left(\boldsymbol{H}_{\boldsymbol{J}}+\beta \operatorname{curl} \boldsymbol{H}_{\boldsymbol{J}}\right)
\end{array}\right)
$$

and introducing the operators

$$
\mathcal{A}_{\beta}=\left(\begin{array}{cc}
A_{\beta} & 0 \\
0 & A_{\beta}
\end{array}\right) \text { and } \mathcal{C}=\left(\begin{array}{cc}
0 & \varepsilon^{-1} \text { curl } \\
-\mu^{-1} \text { curl } & 0
\end{array}\right) .
$$

Assuming that condition (3.11) is satisfied in the remainder of this section, Corollary 3.10 enables us to prove that the above problem is well-posed under the proportionality conditions (1.3). Indeed, considering the new unknown $^{2} \mathscr{D}=\mathcal{A}_{\beta} \mathscr{E}$, problem (4.1) is equivalent to

$$
\left\{\begin{array}{l}
\frac{\mathrm{d} \mathscr{D}}{\mathrm{d} t}-\mathcal{C A}_{\beta}{ }^{-1} \mathscr{D}=\mathscr{F}, t>0, \\
\mathscr{D}(0)=\mathcal{A}_{\beta} \mathscr{E}_{0} .
\end{array}\right.
$$

Let us then denote by $S$ the largest vector subspace of $R\left(A_{\beta}\right)$ such that

$$
A_{\beta}{ }^{-1} S \subset S,
$$

in the sense that

$$
\left\{A_{\beta}{ }^{-1} \boldsymbol{y} \mid \boldsymbol{y} \in S\right\} \subset S .
$$

We now simply remark that the linear operator $\mathcal{C A}_{\beta}{ }^{-1}$ is a bounded operator from $\mathcal{S}=S \times S$ into itself provided (1.3) holds. Indeed, from our assumption on the coefficients, we may write, for any $\left(\begin{array}{c}\varphi \\ \psi\end{array}\right)$ in $\mathcal{S}$,

$$
\begin{aligned}
\mathcal{C A}_{\beta}{ }^{-1}\left(\begin{array}{c}
\boldsymbol{\varphi} \\
\boldsymbol{\psi}
\end{array}\right) & =\left(\begin{array}{c}
\varepsilon^{-1} \operatorname{curl}\left(A_{\beta}{ }^{-1} \boldsymbol{\psi}\right) \\
-\mu^{-1} \operatorname{curl}\left(A_{\beta}{ }^{-1} \boldsymbol{\varphi}\right)
\end{array}\right) \\
& =\left(\begin{array}{c}
\varepsilon^{-1} \beta^{-1} \beta \operatorname{curl}\left(A_{\beta}{ }^{-1} \boldsymbol{\psi}\right) \\
-\mu^{-1} \beta^{-1} \beta \operatorname{curl}\left(A_{\beta}{ }^{-1} \boldsymbol{\varphi}\right)
\end{array}\right) \\
& =\left(\begin{array}{c}
\kappa_{\varepsilon} \beta \operatorname{curl}\left(A_{\beta}{ }^{-1} \boldsymbol{\psi}\right) \\
-\kappa_{\mu} \beta \operatorname{curl}\left(A_{\beta}{ }^{-1} \boldsymbol{\varphi}\right)
\end{array}\right) \\
& =\left(\begin{array}{c}
\kappa_{\varepsilon}\left((I d+\beta \operatorname{curl})\left(A_{\beta}{ }^{-1} \boldsymbol{\psi}\right)-A_{\beta}{ }^{-1} \boldsymbol{\psi}\right) \\
-\kappa_{\mu}\left((I d+\beta \operatorname{curl})\left(A_{\beta}{ }^{-1} \boldsymbol{\varphi}\right)-A_{\beta}{ }^{-1} \boldsymbol{\varphi}\right)
\end{array}\right) \\
& =\left(\begin{array}{c}
\kappa_{\varepsilon}\left(\boldsymbol{\psi}-A_{\beta}{ }^{-1} \boldsymbol{\psi}\right) \\
-\kappa_{\mu}\left(\boldsymbol{\varphi}-A_{\beta}{ }^{-1} \boldsymbol{\varphi}\right)
\end{array}\right) .
\end{aligned}
$$

Hence system $(4.2)$ has a unique mild solution $\mathscr{D} \in \mathscr{C}([0, \infty) ; \mathcal{S})$, for any $\mathcal{A}_{\beta} \mathscr{E} 0 \in \mathcal{S}$ and $\mathscr{F} \in L_{\text {loc }}^{1}(0, \infty ; \mathcal{S})$ (see [12] for instance). Coming back to the original unknowns $\boldsymbol{E}$ and $\boldsymbol{H}$, we have obtained the following result.

TheOREM 4.1. Assume that $\boldsymbol{E}_{0} \in A_{\beta}{ }^{-1} S, \boldsymbol{H}_{0} \in \boldsymbol{H}_{\boldsymbol{J}}(0)+A_{\beta}{ }^{-1} S$, and $\boldsymbol{J}$ is such that there exists a lifting $\boldsymbol{H}_{\boldsymbol{J}} \in W_{\text {loc }}^{1,1}(0, \infty ; H(\beta \operatorname{curl} ; S))$, where $H(\beta \operatorname{curl} ; S)=\{\boldsymbol{v} \in S \mid \beta \operatorname{curl} \boldsymbol{v} \in S\}$. Then, under conditions (1.3) and (3.11), problem (1.1)-(1.2) has a unique solution

$$
\boldsymbol{E} \in \mathscr{C}\left([0, \infty) ; A_{\beta}{ }^{-1} S\right), \boldsymbol{H} \in \boldsymbol{H}_{\boldsymbol{J}}+\mathscr{C}\left([0, \infty) ; A_{\beta}{ }^{-1} S\right) .
$$

In particular, we have

$$
\boldsymbol{E} \in \mathscr{C}\left([0, \infty) ; X_{N}\left(\Omega, \beta^{-1}\right)\right), \boldsymbol{H} \in \boldsymbol{H}_{\boldsymbol{J}}+\mathscr{C}\left([0, \infty) ; X_{N}\left(\Omega, \beta^{-1}\right)\right)
$$

As an end note, let us emphasize the difference between the present approach to the evolution problem (4.1), which amounts (up to some multiplicative coefficients) to use the fields $\boldsymbol{D}$ and $\boldsymbol{B}$ as the unknowns of the Maxwell problem, and the one followed in [8], which kept the "natural" unknowns $\boldsymbol{E}$ and $\boldsymbol{H}$. From the point of view of electromagnetics, this can be seen as a reinterpretation of the original problem, by inversion of the constitutive relations. While, in retrospect, the problem in $\boldsymbol{E}$ and $\boldsymbol{H}$ did not allow us to establish a full existence result, the problem in $\boldsymbol{D}$ and $\boldsymbol{B}$ does not yield directly an explicit functional setting for the data and the solution. So, this new result requires further studies, which are provided in the next section.

\footnotetext{
${ }^{2}$ In other words, from the point of view of electromagnetics, the unknowns are now the fields $\varepsilon^{-1} \boldsymbol{D}$ and $\mu^{-1} \boldsymbol{B}$.
} 
5. Range of applicability of Theorem 4.1. Gathered in this last section are some results intended to make the space $S$ more "explicit". We thus provide some information on the validity of the existence result of the time-dependent solutions in [8].

\subsection{Some elementary results.} $[8])$.

- The vector space $S$ is not reduced to $\{0\}$ (this result has already been alluded to in the Erratum to

Lemma 5.1. Consider the space

$$
S_{0}=\left\{\boldsymbol{\nabla} v \mid v \in H_{c}^{1}(\Omega) \text { and } \operatorname{div}\left(\beta^{-1} \nabla v\right) \in L^{2}(\Omega)\right\} .
$$

Then, $S_{0}$ is a subspace of $R\left(A_{\beta}\right)$ and furthermore

$$
S_{0} \subset S
$$

Proof. For the first assertion, let $\boldsymbol{w} \in R\left(A_{\beta}\right)^{\perp}$ and $\boldsymbol{\nabla} u \in S_{0}$. By the Green formula, we have

$$
(\boldsymbol{\nabla} u, \boldsymbol{w})_{H}=(\boldsymbol{\nabla} u, \boldsymbol{w})_{\beta^{-1}}=0,
$$

since $\operatorname{div}\left(\beta^{-1} \boldsymbol{w}\right)=0$ in $\Omega$, according to Theorem 3.5.

For the second one, we notice that

$$
A_{\beta}{ }^{-1}(\nabla u)=\nabla u,
$$

which belongs to $X_{N}\left(\Omega, \beta^{-1}\right)$. This shows that $A_{\beta}{ }^{-1} S_{0}=S_{0}$, hence the inclusion $S_{0} \subset S$.

- The vector space $S$ is closed.

Lemma 5.2. The vector space $S$ is a closed subspace of $R\left(A_{\beta}\right)$.

Proof. Let $\boldsymbol{x}$ belong to the closure $\bar{S}$, then there exists a sequence $\left(\boldsymbol{x}_{n}\right)_{n \in \mathbb{N}}$ of elements of $S$ going to $\boldsymbol{x}$ in $H\left(\operatorname{div} \beta^{-1} ; \Omega\right)$ as $n$ tends to infinity. Since the embedding of $X_{N}\left(\Omega, \beta^{-1}\right)$ into $H\left(\operatorname{div} \beta^{-1} ; \Omega\right)$ is continuous, as is the operator $A_{\beta}{ }^{-1}$ from $R\left(A_{\beta}\right)$ to $X_{N}\left(\Omega, \beta^{-1}\right)$, we have

$$
A_{\beta}{ }^{-1} \boldsymbol{x}_{n} \rightarrow A_{\beta}{ }^{-1} \boldsymbol{x} \text { in } H \text { as } n \rightarrow \infty \text {. }
$$

This implies that $A_{\beta}{ }^{-1} \boldsymbol{x} \in \bar{S}$ because $\left(A_{\beta}{ }^{-1} \boldsymbol{x}_{n}\right)_{n \in \mathbb{N}}$ is a sequence of elements of $S$.

We just have shown that $A_{\beta}{ }^{-1} \bar{S} \subset \bar{S}$ and consequently that $\bar{S} \subset S$, by definition of $S$. $\square$

\section{- An abstract, but somewhat natural, characterization of $S$.}

Lemma 5.3. The set $S$ is equal to

$$
S=\cap_{k \in \mathbb{N}^{*}} D\left(A_{\beta}{ }^{-k}\right)=\left\{\boldsymbol{y} \in R\left(A_{\beta}\right) \mid \forall k \in \mathbb{N}^{*}, \exists \boldsymbol{z}_{k} \in D\left(A_{\beta}{ }^{k}\right), A_{\beta}{ }^{k} \boldsymbol{z}_{k}=\boldsymbol{y}\right\} .
$$

Proof. Let us set

$$
\breve{S}=\left\{\boldsymbol{y} \in R\left(A_{\beta}\right) \mid \forall k \in \mathbb{N}^{*}, \exists \boldsymbol{z}_{k} \in D\left(A_{\beta}{ }^{k}\right), A_{\beta}{ }^{k} \boldsymbol{z}_{k}=\boldsymbol{y}\right\} .
$$

We first show the inclusion $S \subset \breve{S}$. If $\boldsymbol{y} \in S$, then there exists $\boldsymbol{v} \in X_{N}\left(\Omega, \beta^{-1}\right)$ such that

$$
\boldsymbol{y}=A_{\beta} \boldsymbol{v} .
$$

But, by definition, $\boldsymbol{v}$ also belongs to $S$. Therefore there exists $\boldsymbol{v}_{1} \in X_{N}\left(\Omega, \beta^{-1}\right)$ satisfying

$$
\boldsymbol{v}=A_{\beta} \boldsymbol{v}_{1} .
$$

By iterating, we conclude that $\boldsymbol{y} \in \breve{S}$.

Conversely let $\boldsymbol{y} \in \breve{S}$, then there exists $\boldsymbol{z}_{1} \in X_{N}\left(\Omega, \beta^{-1}\right)$ such that

$$
A_{\beta} \boldsymbol{z}_{1}=\boldsymbol{y} .
$$

This implies that $\boldsymbol{z}_{1}$ belongs to $\breve{S}$, because, for all $k \in \mathbb{N}^{*}$, there exists $\boldsymbol{z}_{k} \in D\left(A_{\beta}{ }^{k}\right)$ such that $A_{\beta}{ }^{k} \boldsymbol{z}_{k}=\boldsymbol{y}$. Therefore, we have $\boldsymbol{z}_{1}=A_{\beta}{ }^{-1}\left(A_{\beta}{ }^{k} \boldsymbol{z}_{k}\right)=A_{\beta}{ }^{k-1} \boldsymbol{z}_{k}$. 
5.2. A more involved result. To establish the interesting property that $S$ is strictly larger than $S_{0}$, we first need a number of intermediate results. Following [8], we introduce the following bilinear form on $X_{N}\left(\Omega, \beta^{-1}\right) \times$ $X_{N}\left(\Omega, \beta^{-1}\right)$

$$
a(\boldsymbol{u}, \boldsymbol{v})=(\boldsymbol{u}+\beta \operatorname{curl} \boldsymbol{u}, \boldsymbol{v}+\beta \operatorname{curl} \boldsymbol{v})_{\beta^{-1}}
$$

$$
+\left(\operatorname{div}\left(\beta^{-1} \boldsymbol{u}\right), \operatorname{div}\left(\beta^{-1} \boldsymbol{v}\right)\right)_{L^{2}(\Omega)}+\left(P_{e} \boldsymbol{u}, P_{e} \boldsymbol{v}\right)_{\beta^{-1}} .
$$

Lemma 5.4. Assume that (3.11) holds. Then, the bilinear form defined by (5.1) is coercive, i.e., there exists $\gamma>0$ such that

$$
a(\boldsymbol{u}, \boldsymbol{u}) \geq \gamma\|\boldsymbol{u}\|_{X_{N}\left(\Omega, \beta^{-1}\right)}^{2}, \forall \boldsymbol{u} \in X_{N}\left(\Omega, \beta^{-1}\right) .
$$

Proof. As in [8], we use a contradiction argument. Suppose that (5.2) does not hold. Then, there exists a sequence $\left(\boldsymbol{u}_{n}\right)_{n \in \mathbb{N}}$ in $X_{N}\left(\Omega, \beta^{-1}\right)$ such that

$$
a\left(\boldsymbol{u}_{n}, \boldsymbol{u}_{n}\right) \rightarrow 0 \text { as } n \rightarrow \infty, \text { and }\left\|\boldsymbol{u}_{n}\right\|_{X_{N}\left(\Omega, \beta^{-1}\right)}=1, \forall n \in \mathbb{N} .
$$

The second property implies that the sequence $\left(\boldsymbol{u}_{n}\right)_{n}$ is bounded in $X_{N}\left(\Omega, \beta^{-1}\right)$. Since $X_{N}\left(\Omega, \beta^{-1}\right)$ is compactly embedded into $L^{2}(\Omega)^{3}$, we deduce that there exists a subsequence, still denoted by $\left(\boldsymbol{u}_{n}\right)_{n \in \mathbb{N}}$, and $\boldsymbol{u}$ in $X_{N}\left(\Omega, \beta^{-1}\right)$ such that

$$
\begin{gathered}
\boldsymbol{u}_{n} \rightarrow \boldsymbol{u} \text { weakly in } X_{N}\left(\Omega, \beta^{-1}\right) \text {, as } n \rightarrow \infty, \\
\boldsymbol{u}_{n} \rightarrow \boldsymbol{u} \text { strongly in } L^{2}(\Omega)^{3} \text {, as } n \rightarrow \infty .
\end{gathered}
$$

Now as (5.3) means that

$$
\begin{aligned}
& \boldsymbol{u}_{n}+\beta \text { curl } \boldsymbol{u}_{n} \rightarrow \mathbf{0} \text { strongly in } L^{2}(\Omega)^{3}, \\
& \operatorname{div}\left(\beta^{-1} \boldsymbol{u}_{n}\right) \rightarrow \mathbf{0} \text { strongly in } L^{2}(\Omega), \\
& P_{e} \boldsymbol{u}_{n} \rightarrow \mathbf{0} \text { strongly in } L^{2}(\Omega),
\end{aligned}
$$

we deduce that $\boldsymbol{u}_{n}$ converges strongly to $\boldsymbol{u}$ in $X_{N}\left(\Omega, \beta^{-1}\right)$ and that

$$
\begin{array}{r}
\boldsymbol{u}+\beta \operatorname{curl} \boldsymbol{u}=\mathbf{0} \text { in } \Omega, \\
\operatorname{div}\left(\beta^{-1} \boldsymbol{u}\right)=0 \text { in } \Omega, \\
P_{e} \boldsymbol{u}=\mathbf{0}, \\
\|\boldsymbol{u}\|_{X_{N}\left(\Omega, \beta^{-1}\right)}=1 .
\end{array}
$$

As $\boldsymbol{u} \wedge \boldsymbol{n}=\mathbf{0}$ on $\Gamma$, the field $\boldsymbol{j}=\beta \operatorname{curl} \boldsymbol{u}$, which belongs to $H\left(\operatorname{div} \beta^{-1} ; \Omega\right)$, satisfies $\boldsymbol{j} \cdot \boldsymbol{n}=0$ on $\Gamma$. This implies that $\boldsymbol{u}=K_{\beta}{ }^{*} \boldsymbol{j}$, according to (3.5) and (3.6). Now using (5.4), we see that

$$
K_{\beta}{ }^{*} \boldsymbol{j}+\boldsymbol{j}=\mathbf{0},
$$

and since the assumption (3.11) guarantees that $I d+K_{\beta}{ }^{*}$ is an isomorphism, we deduce that $\boldsymbol{j}=\mathbf{0}$. Then, one has $\boldsymbol{u}=\mathbf{0}$, which contradicts (5.7).

Lemma 5.5. Assume that (3.11) holds. For any $\boldsymbol{y}$ in $H\left(\operatorname{div} \beta^{-1} 0, \Omega\right) \cap R\left(A_{\beta}\right)$, there exists $\boldsymbol{z} \in D\left(A_{\beta}{ }^{2}\right)$ such that

$$
A_{\beta}^{2} \boldsymbol{z}=\boldsymbol{y}
$$

Proof. As a consequence of Lemma 5.4 and Lax-Milgram's lemma, there exists a unique solution $\boldsymbol{z} \in$ $X_{N}\left(\Omega, \beta^{-1}\right)$ to

$$
a(\boldsymbol{z}, \boldsymbol{v})=(\boldsymbol{y}, \boldsymbol{v})_{\beta^{-1}}+\left(P_{e} \boldsymbol{y}, P_{e} \boldsymbol{v}\right)_{\beta^{-1}}, \forall \boldsymbol{v} \in X_{N}\left(\Omega, \beta^{-1}\right) .
$$

First, choosing $\boldsymbol{v} \in \mathcal{H}\left(e, \beta^{-1}\right)$ yields

$$
\left(P_{e} \boldsymbol{z}, P_{e} \boldsymbol{v}\right)_{\beta^{-1}}=\left(P_{e} \boldsymbol{y}, P_{e} \boldsymbol{v}\right)_{\beta^{-1}}, \forall \boldsymbol{v} \in \mathcal{H}\left(e, \beta^{-1}\right),
$$


and thus

$$
P_{e} \boldsymbol{z}=P_{e} \boldsymbol{y}
$$

Next, for any $g \in L^{2}(\Omega)$, let $\varphi \in H_{0}^{1}(\Omega)$ be the unique solution to (3.9) and consider in (5.8) a test function of the form $\boldsymbol{v}=\boldsymbol{\nabla} \varphi$ to obtain

$$
(\boldsymbol{z}+\beta \operatorname{curl} \boldsymbol{z}, \boldsymbol{\nabla} \varphi)_{\beta^{-1}}+\left(\operatorname{div}\left(\beta^{-1} \boldsymbol{z}\right), \operatorname{div}\left(\beta^{-1} \boldsymbol{\nabla} \varphi\right)\right)_{L^{2}(\Omega)}=0 .
$$

Integrating by parts in the first term in the left-hand side leads to

$$
0=\left(\operatorname{div}\left(\beta^{-1} \boldsymbol{z}\right),-\varphi+\operatorname{div}\left(\beta^{-1} \boldsymbol{\nabla} \varphi\right)\right)_{L^{2}(\Omega)}=\left(\operatorname{div}\left(\beta^{-1} \boldsymbol{z}\right), g\right)_{L^{2}(\Omega)}, \forall g \in L^{2}(\Omega),
$$

and therefore

$$
\operatorname{div}\left(\beta^{-1} \boldsymbol{z}\right)=0 \text { in } \Omega
$$

Problem (5.8) then reduces to

$$
(\boldsymbol{z}+\beta \operatorname{curl} \boldsymbol{z}, \boldsymbol{v}+\beta \operatorname{curl} \boldsymbol{v})_{\beta^{-1}}=(\boldsymbol{y}, \boldsymbol{v})_{\beta^{-1}}, \forall \boldsymbol{v} \in X_{N}\left(\Omega, \beta^{-1}\right),
$$

and, choosing $\boldsymbol{v}$ in $\mathscr{D}\left(\Omega_{k}\right)$ for $1 \leq k \leq K$, we see that (5.10) implies that

$$
(I d+\beta \operatorname{curl})(\boldsymbol{z}+\beta \operatorname{curl} \boldsymbol{z})=\boldsymbol{y} \text { in } \Omega_{k} .
$$

Hence, the field $\boldsymbol{z}+\beta \operatorname{curl} \boldsymbol{z}$ belongs to $H\left(\operatorname{curl}, \Omega_{k}\right)$. Returning to (5.10) and applying the Green formula on each subdomain $\Omega_{k}$, we find

$$
\sum_{k=1}^{K}\langle(\boldsymbol{z}+\beta \operatorname{curl} \boldsymbol{z}) \wedge \boldsymbol{n}, \boldsymbol{n} \wedge(\boldsymbol{v} \wedge \boldsymbol{n})\rangle_{\partial \Omega_{k}}=0, \forall \boldsymbol{v} \in X_{N}\left(\Omega, \beta^{-1}\right) .
$$

This implies that $\boldsymbol{z}+\beta \operatorname{curl} \boldsymbol{z}$ belongs to $H(\operatorname{curl}, \Omega)$ and satisfies

$$
(I d+\beta \operatorname{curl})(\boldsymbol{z}+\beta \operatorname{curl} \boldsymbol{z})=\boldsymbol{y} \text { in } \Omega .
$$

It remains to check that

$$
(\boldsymbol{z}+\beta \operatorname{curl} \boldsymbol{z}) \wedge \boldsymbol{n}=0 \text { on } \Gamma \text {. }
$$

This boundary condition cannot be deduced from (5.11) because the test functions $\boldsymbol{v}$ are in $X_{N}\left(\Omega, \beta^{-1}\right)$. But, noticing that $\boldsymbol{y}$ belongs to $R\left(A_{\beta}\right)$, it stems from (5.12) that $(I d+\beta$ curl $)(\boldsymbol{z}+\beta \operatorname{curl} \boldsymbol{z})$ is in $R\left(A_{\beta}\right)$ as well, or, equivalently, is orthogonal to $R\left(A_{\beta}\right)^{\perp}$ in $H\left(\operatorname{div} \beta^{-1} ; \Omega\right)$, which yields, keeping (5.9) in mind,

$$
((I d+\beta \operatorname{curl})(\boldsymbol{z}+\beta \operatorname{curl} \boldsymbol{z}), \boldsymbol{w})_{\beta^{-1}}=0, \forall \boldsymbol{w} \in R\left(A_{\beta}\right)^{\perp} .
$$

After an integration by parts, we obtain

$$
(\boldsymbol{z}+\beta \operatorname{curl} \boldsymbol{z}, \boldsymbol{w}+\beta \operatorname{curl} \boldsymbol{w})_{\beta^{-1}}+\langle\boldsymbol{w} \wedge \boldsymbol{n}, \boldsymbol{n} \wedge((\boldsymbol{z}+\beta \operatorname{curl} \boldsymbol{z}) \wedge \boldsymbol{n})\rangle_{\Gamma}=0, \forall \boldsymbol{w} \in R\left(A_{\beta}\right)^{\perp},
$$

and, owing to Theorem 3.5,

$$
\langle\boldsymbol{w} \wedge \boldsymbol{n}, \boldsymbol{n} \wedge((\boldsymbol{z}+\beta \operatorname{curl} \boldsymbol{z}) \wedge \boldsymbol{n})\rangle_{\Gamma}=0, \forall \boldsymbol{w} \in R\left(A_{\beta}\right)^{\perp} .
$$

We finally deduce from the results of Lemma 3.11 that

$$
\boldsymbol{n} \wedge((\boldsymbol{z}+\beta \operatorname{curl} \boldsymbol{z}) \wedge \boldsymbol{n})=0 \text { in } H^{-1 / 2}\left(\operatorname{curl}_{\Gamma} ; \Gamma\right),
$$

which implies (5.13). $\Omega$.

LEMmA 5.6. Let $\boldsymbol{y} \in S$ be such that it is orthogonal to $S_{0}$ in $H\left(\operatorname{div} \beta^{-1} ; \Omega\right)$. Then, one has $\operatorname{div}\left(\beta^{-1} \boldsymbol{y}\right)=0$ in 
Proof. The orthogonality assumption is equivalent to

$$
\int_{\Omega}\left(\beta^{-1} \boldsymbol{y} \cdot \nabla u+\operatorname{div}\left(\beta^{-1} \boldsymbol{y}\right) \operatorname{div}\left(\beta^{-1} \nabla u\right)\right) \mathrm{d} \boldsymbol{x}=0,
$$

$$
\forall u \in\left\{v \in H_{0}^{1}(\Omega) \mid \operatorname{div}\left(\beta^{-1} \nabla v\right) \in L^{2}(\Omega)\right\} .
$$

Once more, for any $g \in L^{2}(\Omega)$, we consider $\varphi \in H_{0}^{1}(\Omega)$ the unique solution to (3.9) and take $u=\varphi$ hereabove. Applying the Green formula then leads to

$$
\int_{\Omega} \operatorname{div}\left(\beta^{-1} \boldsymbol{y}\right) g \mathrm{~d} \boldsymbol{x}=0, \forall g \in L^{2}(\Omega)
$$

hence the conclusion. $\square$

\section{- The vector space $S$ is larger than $S_{0}$.}

Lemma 5.7. The set $S \backslash S_{0}$ is non empty.

Proof. Let $\boldsymbol{y} \in S \cap S_{0}^{\perp}$. Then, by virtue of Lemma 5.6, the field $\beta^{-1} \boldsymbol{y}$ is divergence-free and therefore, owing to Lemma 3.3, we have the following decomposition

$$
\boldsymbol{y}=\boldsymbol{\nabla} s+\sum_{j=1}^{m} c_{j} \nabla q_{j}^{T}+\beta \operatorname{curl} \phi
$$

with $s \in H^{1}(\Omega) / \mathbb{R}$, coefficients $c_{j}, 1 \leq j \leq m$, and $\boldsymbol{\phi} \in X_{N}\left(\Omega, \beta^{-1}\right)$, such that $\operatorname{div}\left(\beta^{-1} \boldsymbol{\phi}\right)=0$ in $\Omega$ and $P_{e} \boldsymbol{\phi}=\mathbf{0}$. Since $\boldsymbol{y}$ belongs to $S$, it is orthogonal to $R\left(A_{\beta}\right)^{\perp}$ and therefore

$$
(\boldsymbol{y}, \boldsymbol{w})_{H}=0, \forall \boldsymbol{w} \in R\left(A_{\beta}\right)^{\perp}
$$

or equivalently, using the above decomposition of $\boldsymbol{y}$,

$$
\int_{\Omega}\left(\beta^{-1} \nabla s+\sum_{j=1}^{m} c_{j} \beta^{-1} \nabla q_{j}^{T}+\operatorname{curl} \phi\right) \cdot \boldsymbol{w} \mathrm{d} \boldsymbol{x}=0, \forall \boldsymbol{w} \in R\left(A_{\beta}\right)^{\perp} .
$$

Applying the Green formula (bearing in mind that $\boldsymbol{w}+\beta \operatorname{curl} \boldsymbol{w}=\mathbf{0}$ and that $\operatorname{div}\left(\beta^{-1} \boldsymbol{w}\right)=0$ in $\Omega$ ), we obtain

$$
\left\langle\beta^{-1} \boldsymbol{w} \cdot \boldsymbol{n}, s+\sum_{j=1}^{m} c_{j} q_{j}^{T}\right\rangle_{\Gamma}-(\boldsymbol{\phi}, \boldsymbol{w})_{\beta^{-1}}=0, \forall \boldsymbol{w} \in R\left(A_{\beta}\right)^{\perp} .
$$

Therefore, using Lemma 3.6, we deduce that

$$
\left\langle g, s+\sum_{j=1}^{m} c_{j} q_{j}^{T}\right\rangle_{\Gamma}-(\boldsymbol{\phi}, \boldsymbol{w})_{\beta^{-1}}=0, \forall g \in H_{0}^{-1 / 2}(\Gamma),
$$

with $\boldsymbol{w}$ given as in Lemma 3.6. Let us next fix $\widetilde{\boldsymbol{\phi}} \in X_{N}\left(\Omega, \beta^{-1}\right)$, such that $\operatorname{div} \beta^{-1} \widetilde{\boldsymbol{\phi}}=0$ and $P_{e} \widetilde{\boldsymbol{\phi}}=\mathbf{0}$, and consider the unique $s_{0} \in H^{1 / 2}(\Gamma) / \mathbb{R}$ governed by

$$
\left\langle s_{0}, g\right\rangle_{\Gamma}=(\widetilde{\boldsymbol{\phi}}, \boldsymbol{w})_{\beta^{-1}}, \forall g \in H_{0}^{-1 / 2}(\Gamma) .
$$

Such a $s_{0}$ exists because the right-hand side of this identity is a linear form on $H_{0}^{-1 / 2}(\Gamma)$. Now consider the unique lifting $\widetilde{s} \in H^{1}(\Omega) / \mathbb{R}$ such that

$$
\begin{aligned}
& \operatorname{div}\left(\beta^{-1} \nabla \widetilde{s}\right)=0 \text { in } \Omega, \\
& \widetilde{s}=s_{0} \text { on } \Gamma .
\end{aligned}
$$

In view of (5.14), the property (5.15) implies that the field $\widetilde{\boldsymbol{y}}$, defined by

$$
\widetilde{\boldsymbol{y}}=\nabla \widetilde{s}+\beta \operatorname{curl} \widetilde{\phi}
$$

is orthogonal to $R\left(A_{\beta}\right)^{\perp}$. This means that $\widetilde{\boldsymbol{y}}$ belongs to $R\left(A_{\beta}\right)$. Moreover, by construction, the field $\beta^{-1} \widetilde{\boldsymbol{y}}$ is divergence-free (and, as such, it is orthogonal to $S_{0}$ ). Hence, by virtue of Lemma 5.5, there exists $\boldsymbol{z} \in D\left(A_{\beta}{ }^{2}\right)$ such that

$$
A_{\beta}^{2} \boldsymbol{z}=\widetilde{\boldsymbol{y}}
$$


Now let $\boldsymbol{v} \in X_{N}\left(\Omega, \beta^{-1}\right)$ be such that $\widetilde{\boldsymbol{y}}=A_{\beta} \boldsymbol{v}$, then we remark that

$$
A_{\beta}\left(A_{\beta} \boldsymbol{z}-\boldsymbol{v}\right)=0
$$

and since $A_{\beta}$ is injective we deduce that

$$
\boldsymbol{v}=A_{\beta} \boldsymbol{z}
$$

which garantees that $\boldsymbol{v}$ belongs to $R\left(A_{\beta}\right)$. Moreover, since

$$
\widetilde{\boldsymbol{y}}=\boldsymbol{v}+\beta \operatorname{curl} \boldsymbol{v},
$$

and $\beta^{-1} \widetilde{\boldsymbol{y}}$ is divergence-free, we deduce that $\beta^{-1} \boldsymbol{v}$ is divergence-free. Applying once more Lemma 5.5 , there exists $z_{3} \in D\left(A_{\beta}^{2}\right)$ such that

$$
A_{\beta}^{2} \boldsymbol{z}_{3}=\boldsymbol{v}
$$

which implies that

$$
A_{\beta}{ }^{3} z_{3}=A_{\beta} \boldsymbol{v}=\widetilde{\boldsymbol{y}}
$$

By iteration, we show that $\widetilde{\boldsymbol{y}}$ belongs to $D\left(A_{\beta}{ }^{k}\right)$, for all $k \in \mathbb{N}$, and we conclude using Lemma 5.3.

\section{REFERENCES}

[1] H. Ammari and G. BaO, Maxwell's equations in periodic chiral structures, Math. Nachr., 251 (2002), pp. 3-18.

[2] C. Amrouche, C. Bernardi, M. Dauge, And V. Girault, Vector potentials in three-dimensional nonsmooth domains, Math. Methods Appl. Sci., 21 (1998), pp. 823-864.

[3] C. Athanasiadis, G. Costakis, and I. G. Stratis, Electromagnetic scattering by a perfectly conducting obstacle in a homogeneous chiral environment: solvability and low-frequency theory, Math. Methods Appl. Sci., 25 (2002), pp. $927-944$.

[4] A.-S. Bonnet-Ben Dhia, P. Ciarlet, Jr., And C. M. ZwölF, A new compactness result for electromagnetic waves. Application to the transmission problem between dielectrics and metamaterials, Math. Models Methods Appl. Sci., 18 (2008), pp. 16051631 .

[5] T.-Z. Boulmezaoud, Y. MADAY, AND T. Amari, On the linear Beltrami fields in bounded and unbounded three-dimensional domains, ESAIM Math. Model. Numer. Anal., 33 (1999), pp. 359-393.

[6] A. Buffa And P. Ciarlet, JR., On traces for functional spaces related to Maxwell's equations. Part I: an integration by parts formula in Lipschitz polyhedra, Math. Methods Appl. Sci., 24 (2001), pp. 9-30.

[7] A. Buffa, M. Costabel, And D. Sheen, On traces for H(curl, $\Omega$ ) in Lipschitz domains, J. Math. Anal. Appl., 276 (2002), pp. $845-867$.

[8] P. Ciarlet, JR. And G. Legendre, Well-posedness of the Drude-Born-Fedorov model for chiral media, Math. Models Methods Appl. Sci., 17 (2007), pp. 461-484. Erratum, Math. Models Methods Appl. Sci., 19 (2009), pp. $173-174$.

[9] D. J. Frantzeskakis, A. Ionnnidis, G. F. Roach, I. G. Stratis, And A. N. Yannacopoulos, On the error of the optical response approximation in chiral media, Appl. Anal., 82 (2003), pp. 839-856.

[10] A. LAKhtakia, Beltrami fields in chiral media, vol. 2 of World Scientific series in contemporary chemical physics, World Scientific, Singapore, 1994.

[11] K. B. Liaskos, I. G. Stratis, And A. N. Yannacopoulos, A priori estimates for a singular limit approximation of the constitutive laws for chiral media in the time domain, Abstr. Appl. Anal., 355 (2009), pp. $288-302$.

[12] A. PAZY, Semigroups of linear operators and applications to partial differential equations, vol. 44 of Applied mathematical sciences, Springer-Verlag, New York, 1983.

[13] C. Weber, A local compactness theorem for Maxwell's equations, Math. Methods Appl. Sci., 2 (1980), pp. 12-25.

[14] Z. Yoshida And Y. Giga, Remarks on spectra of operator Rot, Math. Z., 204 (1990), pp. 235-245. 